\title{
REPERTORIO LATINOAMERICANO PARA CLARINETE, VIOLA Y PIANO: CATÁLOGO CON COMENTARIOS
}

LATIN AMERICAN REPERTOIRE FOR CLARINET, VIOLA AND PIANO:

CATALOG WITH COMMENTS

Fecha de recibido: 06/07/2018

Fecha de aceptado: 25/10/2018

Karen Johana Correa Suárez*

Tutora:

Profesora Braunwin Sheldrick, DMA

DOI: 10.17230/ricercare.2018.12.3

Pintura en la portada: Caminos del viento, por

Carlos Jacanamijoy

* Estudios de pregrado en la Universidad Francisco José de Caldas. Estudios de maestría en la Universidad EAFIT. Ganadora de la beca de investigación 2017 de la Universidad EAFIT con la investigación cuyos resultados se exponen en el presente artículo. 


\section{Resumen}

Este artículo tiene como objetivo proporcionar una contribución para el reconocimiento del nuevo repertorio de música de cámara latinoamericano para clarinete, viola y piano. La naturaleza de este documento no pretende ser musicológica o teórica, sino, más bien, una organización de repertorio existente para este conjunto. Para tal fin, las principales fuentes consultadas fueron compositores latinoamericanos referenciados en páginas web personales e institucionales, entrevistas vía correo electrónico y catálogos de referencia. Se compiló una lista de obras, así como una revisión de su estética y las experiencias de los compositores. Se espera que este trabajo facilite posteriores puestas en escena, grabaciones y análisis de estas nuevas obras en el campo de la música académica y en la escena cultural mundial.

Palabras clave: Clarinete, música latinoamericana, música de cámara, piano, trío, viola.

\section{Abstract}

This article aims to provide a contribution toward the acknowledgement of new Latin American chamber music repertoire for clarinet, viola, and piano. The nature of this document is not intended to be musicological or theoretical, but rather, an organization of extant repertoire for this ensemble. To this end, the main sources consulted were Latin American composers referenced in personal and institutional web pages, interviews via email and reference catalogs. A list of works was compiled, as well as a review of their aesthetics, and the experiences of composers. This work will hopefully facilitate subsequent performances, recordings, and analyses of these new works within the field of academic music and the global cultural scene.

Key words: Clarinet, chamber music, Latin-American music, piano, trio ensemble, viola. 


\section{Introducción}

La innovación musical es una búsqueda constante, las nuevas ideas, las nuevas formaciones de conjuntos instrumentales, las puestas en escena y la interdisciplinaridad hacen que se nutran nuestras miradas y nuestras experiencias musicales en la actualidad. También, esta nueva oportunidad de conocer repertorios nuevos permite que nos alejemos de los conjuntos apreciados a lo largo de la tradición en los ámbitos cultural y académico, como lo han sido los cuartetos, los dúos con piano, por ejemplo, y la interpretación de música de cámara anterior al siglo XX.

Es así como nació el interés por conjuntos como el conformado por clarinete, viola y piano, que nos permite apreciar las bondades de cada instrumento, como es el caso del clarinete, versátil, siempre expresivo, oscuro y brillante a la vez, que contrasta con la mezcla especial que puede lograr con la viola, que, entre los instrumentos con arco, posee una tesitura especial, no es brillante, pero tampoco oscura, siempre pastosa y cálida. Por otra parte, el piano une con gran contundencia los instrumentos melódicos y nos presenta muchas más posibilidades de explotación sonora mediante el uso de elementos percutidos en su caja de resonancia, o directamente en las cuerdas, registros extremos y colores únicos.

El camino que se siguió para encontrar los datos consignados en el presente artículo fue el contacto directo con las fuentes creadoras, que permitieron un mayor entendimiento de las obras y la oportuna consulta de nuestras dudas con respecto a sus propuestas musicales y estéticas. Se presenta, entonces, un listado de las obras encontradas, con comentarios que describen los requerimientos técnicos que cada una exige y, además, datos básicos como las fechas de creación, estreno y referencias en audio o en video sobre cada una --si existen--, así como una corta descripción de la forma en la que están escritas, para comprensión e inquietud de los interesados en consultar dicho repertorio y apropiarse de él.

\section{El conjunto: clarinete, viola y piano}

Se han presentado varias investigaciones y algunas disertaciones sobre el repertorio latinoamericano para viola (Stephenson, 2011; Vdóvina Vdóvina, 2006; Montano, 2016), lo mismo que para piano ${ }^{1}$ y clarinete por separado (ClariPeru, 2011; Colegio de Compositores Latinoamericanos de Música de Arte, 2012) y el repertorio para piano es el más rico. En el caso de la viola, podemos encontrar documentos en trabajos investigativos, como el catálogo de obras ---en revisión para publicación escrita--del violista venezolano J. Alfonzo, profesor de viola en la Universidad de Stetson (Florida, Estados Unidos), miembro fundador del sistema de orquestas sinfónicas de Venezuela (entrevistas telefónicas y mediante correo electrónico de la autora con el profesor Alfonzo entre 5 de agosto de 2016 y 7 de noviembre de 2017), y el catálogo en línea como fuente referencial de obras latinoamericanas para viola, de esta autora y violista (Correa Suárez, 2013). Estos catálogos están en constante actualización.

1 Se encuentran abundantes textos de tesis, disertaciones e inclusiones en catálogos de compositores que harían extensa la citación, pero pueden ser consultadas en el listado de referencias. 
En el caso de las obras para el conjunto que nos ocupa, algunas han sido encargadas especialmente por ejecutantes en este formato de trío, así como otras se han concebido o transcrito por iniciativa del compositor, con la esperanza de ser llevadas al público con mayor facilidad.

\section{Metodología}

La investigación realizada se basó en un modelo etnográfico fundado en la búsquedá de material musical académico escrito a partir del siglo XIX hasta nuestros días y que esperaba, como primera fuente de búsqueda, hallar datos y archivos en los centros de documentación musical locales o cercanos a Medellín, es decir, Bogotá, Cali, Bucaramanga, Ibagué y Cartagena. Sin embargo, la existencia de archivos que pudieran documentar la existencia de obras para clarinete, viola y piano, escritas originalmente en Latinoamérica --partituras, libros o, por lo menos, artículos de revistas que hablaran sobre el tema, e, incluso, obras o libros sobre los instrumentos considerados en forma individual--es escasa.

Sí se encuentran, de manera específica, libros y partituras de estudios técnicos de cada instrumento por separado, de niveles de aprendizaje desde el básico hasta el profesional en su mayoría. También se consultaron algunas investigaciones en torno al repertorio latinoamericano, que arrojaron diversos estudios desde los puntos de vista sociológico (Miranda y Tello, 2011, Olivarec, 2000) y formativo (Nobre, 1978); desde los niveles iniciales de educación musical hasta los de educación musical superior (Víquez Córdoba, 2011) en los sentidos económico y de la tradición oral y académica (Quintana Moreno, 2005). Todo lo anterior se expuso para así discernir la pertinencia de búsqueda del repertorio que espera describir el presente artículo.

Por otro lado, en los casos de compositores de comienzos del siglo XX, la falta de organización, ya del propio compositor, ya del lugar en el que se salvaguarda o, a veces, por sucesos externos, ha hecho que gran parte de esta música se haya perdido. En algunas situaciones afortunadas, las obras se han digitalizado a partir del año 2000, lo que ha permitido así su circulación en el medio académico. En el caso colombiano, ha sido escaso el resultado de investigación, sumado a que muchas de las obras se encuentran en archivos privados de herencias de los compositores, cuyos curadores no fueron cooperativos con el presente trabajo. Otros optaron por solicitar un respaldo de la institución que cobija la investigación, lo que terminó siendo impráctico.

Debido a las dificultades mencionadas, y una vez se determinó, durante la búsqueda, que el repertorio había sido compuesto en los últimos 30 años, se decidió buscar en las fuentes primarias de creación: los compositores. La consulta a través de páginas web de instituciones musicales alrededor del mundo y de portales web (CEDINIM, 2017), blogs (ClariPeru, 2011; Correa, 2013; Rubio, 2014) y páginas en redes sociales

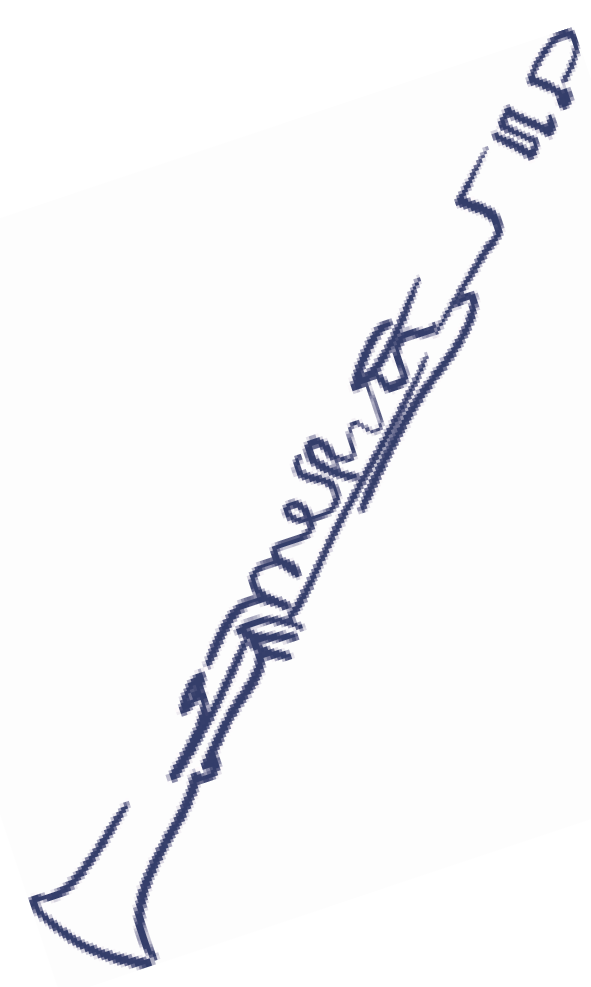


de los compositores y ejecutantes-intérpretes fue relevante ${ }^{2}$. Las tecnologías y las nuevas maneras de comunicarnos de manera más cercana han hecho que FacebookMessenger se haya prestado como herramienta de contacto con maestros dentro de Colombia y en el exterior.

Así fue como una extensa red de creadores musicales aportaron sus opiniones acerca de la presente investigación y también refirieron a colegas dedicados a la escritura musical en diversos formatos. Se consultaron alrededor de setenta y dos compositores de países como Colombia, Argentina, México, Cuba, Costa Rica, Honduras, Nicaragua, Venezuela, Puerto Rico, Paraguay, Uruguay, Brasil, Bolivia y Chile.

Infortunadamente, al consultar a los instrumentistas latinoamericanos y a algunos otros músicos en el mundo, interesados por este repertorio para su respectivo instrumento, no hallamos un conocimiento amplio sobre el repertorio existente para este formato de cámara. Por el contrario, tenemos la esperanza de que este artículo amplíe este conocimiento y anime a su ejecución y reconocimiento.

\section{Resultados y discusión}

Hay factores diversos por considerar en cuanto a este repertorio en la actualidad. La demanda de obras para el formato estudiado no abunda; algunas obras incluyen circunstancias específicas notables como una particular disposición espacial en el escenario. No todos los compositores consultados han escrito obras para este trío, pero se gestaron iniciativas, por parte de algunos compositores, para trascribir o adaptar, o bien, para escribir obras originales con destino a este formato de trío. Desde luego, tales obras, en su mayoría, no han sido grabadas ni estrenadas.

A continuación describo el repertorio encontrado, que suma alrededor de veintiuna obras, con una breve descripción de características específicas estudiadas sobre la partitura, una perspectiva formal muy general y algunos apuntes acerca de las necesidades técnicas propuestas por sus creadores, visto todo ello desde la perspectiva del instrumentista, tal como antes se anotó.

\section{Trioneiron. Gerardo Gandini (Argentina) ${ }^{3}$}

Obra escrita entre 1978 y 1980. Usa un tipo de notación proporcional espacial que indica la duración de las alturas propuestas y motivos para cada uno de los instrumentos. Clarinete en si bemol: la parte del clarinete contiene una serie de alturas y motivos escritos con indicaciones de carácter para cada uno; sin embargo, es de libre elección del intérprete el tiempo que dedique a cada motivo. También, contiene indicaciones de uso de multifónicos en el clarinete. Las frases de cada grupo de alturas e indicaciones se encuentran terminadas con una fermata.

2 La lista de sitios web de los compositores y páginas en Facebook es extensa y por tal motivo se recomienda al lector consultar el listado de referencias en la web en la sección final del artículo.

3 Partitura encontrada en el Latín American Music Center, Universidad de Indiana-Bloomington, Indiana. 


\section{Figura 1. Indicaciones generales de Trioneiron y parte principal de Sol oneiron (parte del clarinete)}

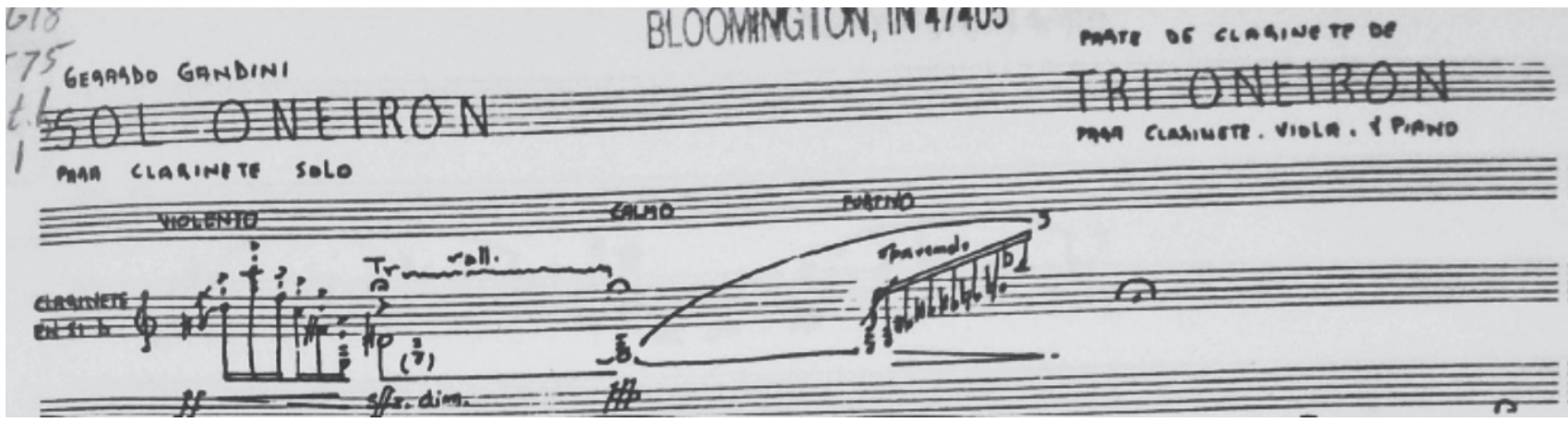

Fuente: Gandini (1978-1980).

La parte del piano, siempre constante en la mano derecha, dado por la indicación inicial que Gandini (1978-1980) señala: dos corcheas con tempo entendido como - = 80 , que sigue una pauta rítmica en corcheas que siempre se mueve por las notas mi 5 , sol\# 5, re\# 5 y re 5 y con indicación exageradamente pppp. Sin embargo, en la mano izquierda se encuentran notas de manera esporádica, que indican articulaciones como trino o arpeggiatos o indicaciones de aceleración de las figuras.

Figura 2. Parte inicial del piano

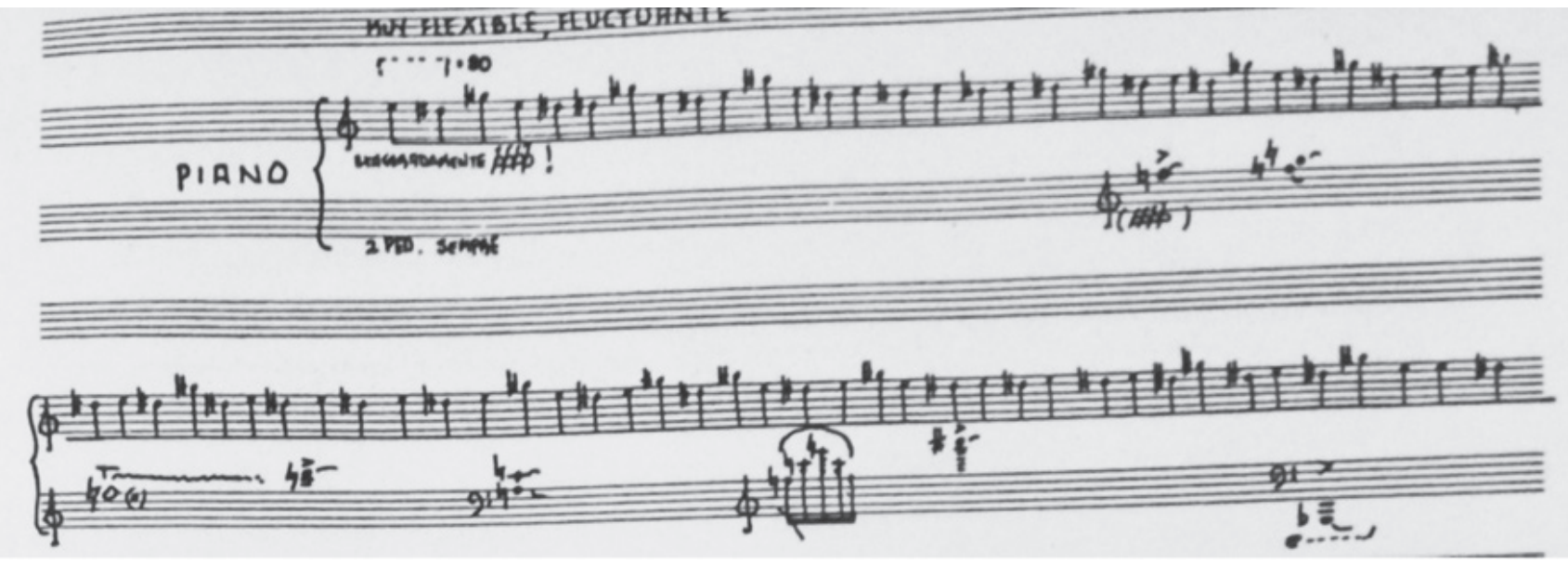

Fuente: Gandini (1978-1980).

La partitura de la viola es la única que tiene un poco más especificadas sus entradas y sus secciones, de acuerdo con los sitios de entradas para los demás instrumentos. Desde el punto de vista técnico para la viola, el uso de puntos de contacto (solos o combinados), como sul tasto, sul ponticello, está presente en toda la partitura, así como indicaciones de pizzicato con la mano izquierda y trinos y armónicos naturales en ciertos puntos climáticos de cada sección. No se mantiene en su registro distintivo 
-bajo el do 3-, salvo la quinta sección, que está en su totalidad en este registro mediobajo. El uso de armónicos naturales y artificiales es recurrente.

Figura 3. Indicaciones iniciales parte de la viola

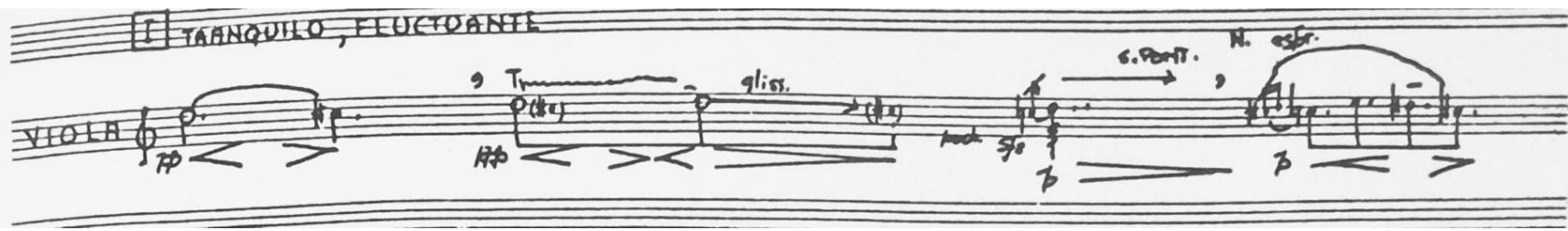

Fuente: Gandini (1978-1980).

Figura 4. Indicaciones específicas en la partitura de la viola

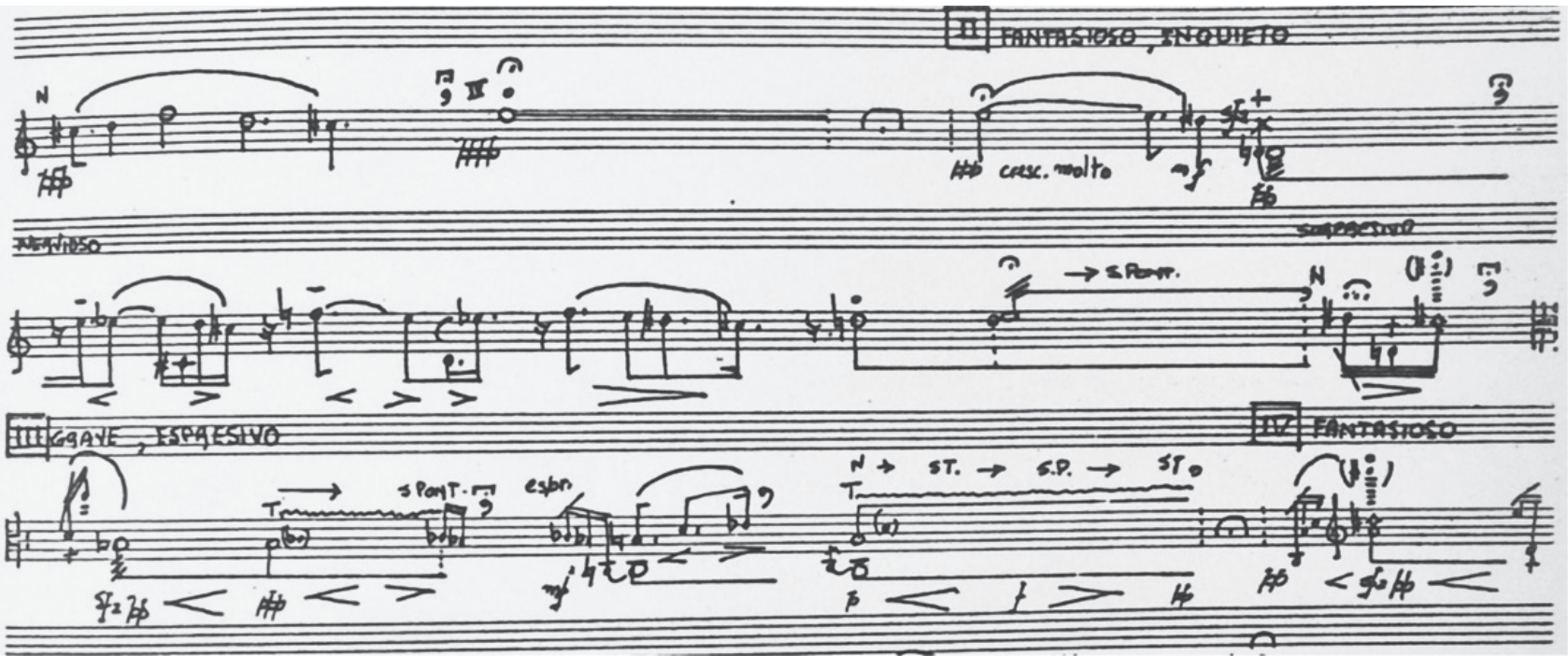

Fuente: Gandini (1978-1980).

\section{Elegía. Luis Arias (Argentina) ${ }^{4}$}

Escrita en 1963, a la memoria de Tirso Olazábal. Especifica la portada que la obra también puede ser interpretada por un violonchelo en lugar de la viola (Arias, 1963). Es una pieza con cambios rítmicos aleatorios en la que predomina la repetición de los compases 2/4,3/4 y 5/8. El uso de relaciones de corchea o de negra con subdivisiones irregulares es frecuente; por ejemplo: $3: 2$, que ha de entenderse como tres negras en duración de dos negras; o 7:8, que significa siete fusas en duración de ocho fusas, etc., con mucho detalle en las dinámicas para cada instrumento. Supone, entonces, una búsqueda de colores entre los tres instrumentos.

La obra se encuentra escrita en sonidos reales para el clarinete. La viola llega a registros extremos mediante el uso de armónicos artificiales y naturales y sonoridades

4 Partitura encontrada en el Latín American Music Center, Universidad de Indiana-Bloomington, Indiana. 
en clave de sol. El piano, por su parte, es el que guía armónicamente la pieza, ya que especifica los cambios cromáticos, incluso como efectos mientras señala pedal.

Figura 5. Indicaciones generales entre compases 4 y 7

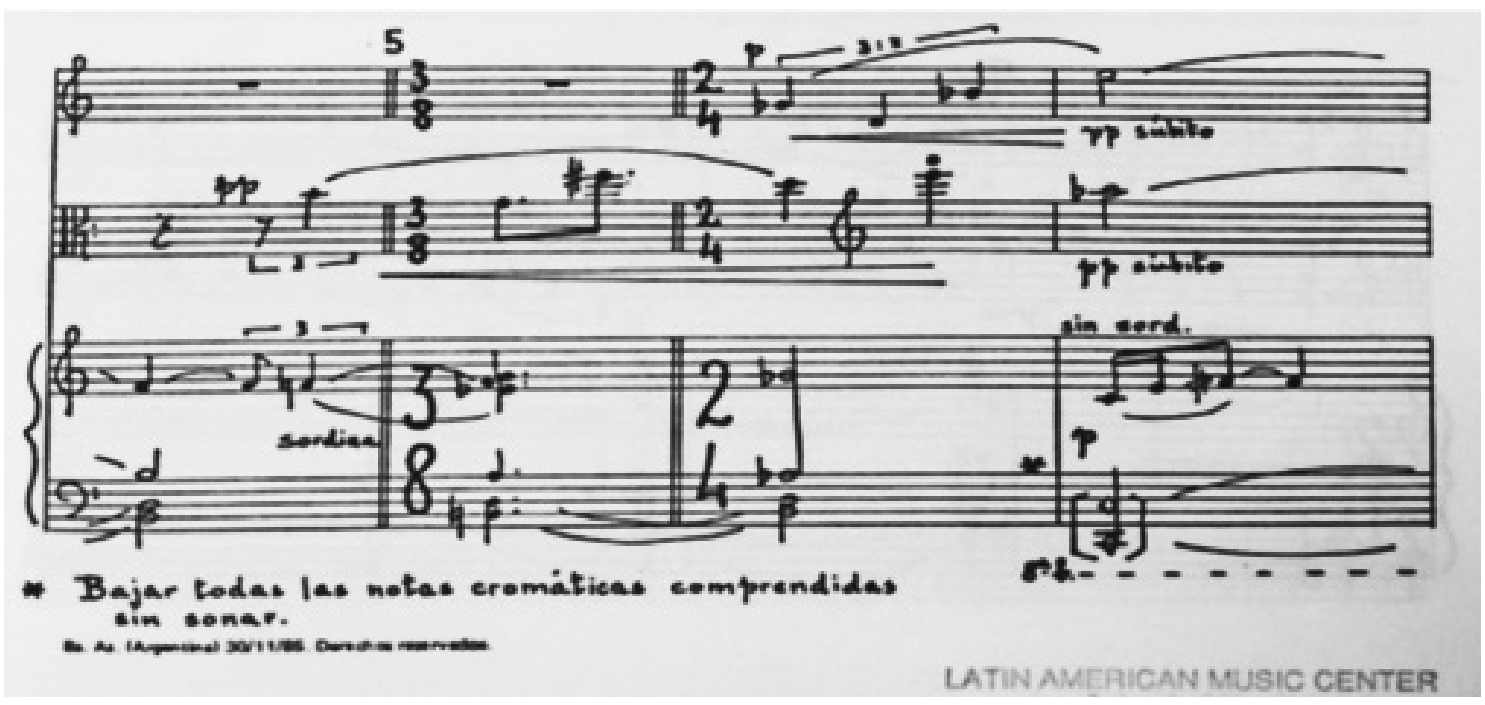

Fuente. Arias (1963).

En general, es una obra que propone colores y su velocidad $(d=40)$ sugiere una composición tranquila y que busca generar atmósferas.

\section{O sabio em sol. Nestor de Hollanda Cavalcanti (Brasil) ${ }^{5}$}

Obra escrita para trío con opción de narrador adicional. Texto de Luiz Guillermo de Beaurepaire (2002). Estrenada por el trío Agosto, integrado por Lucia Morelenbaum (clarinete), Nayran Pessanha (viola) y Josiane Kevorkian (piano) en la XVI Bienal de Música Contemporánea Brasilera, sala Cecilia Maireles, en Río de Janeiro, el 10 de noviembre de 2005.

No se presenta descripción de la partitura, pues no fue posible su consecución.

\section{Trío para clarinete, viola y piano. João Guilherme Ripper (Brasil) ${ }^{6}$}

Obra escrita en 2006, originalmente para el formato clarinete en Bb, viola y piano; y formato opcional de oboe, fagot y piano. Editado por Jeané-Inc Publishing Co.

Sin embargo, la partitura sugiere la posibilidad de ser interpretada por violín en lugar del clarinete.

5 Derechos de autor: Academia Brasilera de Música.

6 Partitura disponible en www.joaoripper.com.br 
Estrenada en Espaço Guiomar Novaes, OPES, serie de cámara Armando Prazeres VII, en Río de Janeiro, el 9 de agosto de 2017, por el trío integrado por los maestros Cristiano Alves (clarinete), Fernando Thebaldi (viola) y Yuka Shimizu (piano).

Figura 6. Inicio de la obra, partitura general (piano)

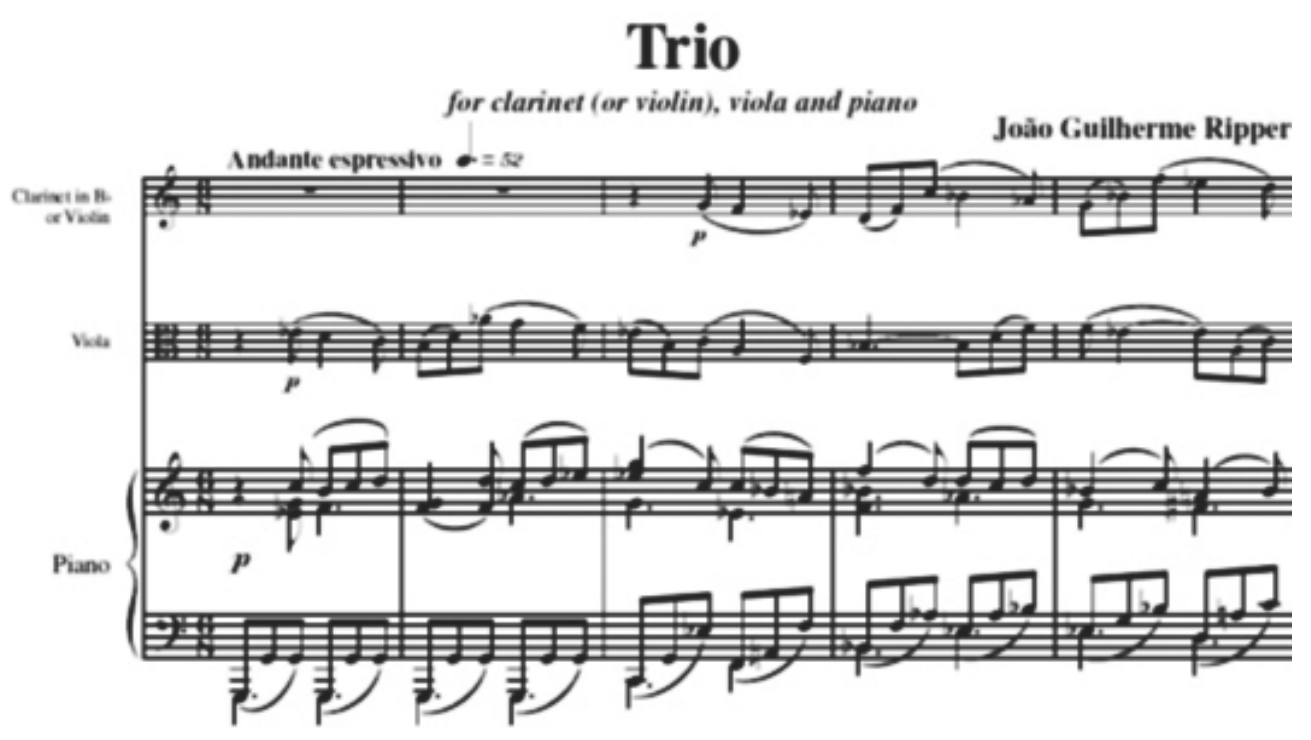

Fuente: Ripper (2007).

La obra está escrita en un movimiento en forma sonata, cuyo primer tema muy lírico contrasta con una danza como segundo tema. Las melodías del clarinete y la viola por lo general se acompañan en contrapunto a la tercera, mientras el piano realiza giros armónicos sobre rítmicas contrastantes. En el sentido técnico, no representa desafíos para los instrumentistas; sin embargo, la variedad de rítmicas y centros tonales exige un gran trabajo de afinación.

\section{Trio das águas. Ricardo Tacuchian (Brasil)}

Obra escrita en 2012. Comisionada por la pianista Ana Carolina Sacco, que la estrenó con motivo de su graduación como magíster en Música. En 2018 se realizará la grabación de un disco compacto dedicado a las obras escritas para viola por el maestro Tacuchian, a cargo del violista brasileño Fernando Thebaldi. Existe un breve escrito para el ensamble brasileño A-trio, en el que el compositor describe su obra (Tacuchian, 2017) y que se resume a continuación:

La música y el agua tienen mucha afinidad...Ambas son flexibles, transparentes, fluidas. No se vive sin agua, como no se vive sin música... Lo que me importa cuando escribo una canción es la construcción bien resuelta de una forma musical, aunque ella parta de una motivación extra musical. Así que podría inspirarme en el agua y escribir una pieza sinfónica o una canción electroacústica. Lo importante es provocar un estímulo para el oyente...El compositor propone, el intérprete realiza

7 Derechos de autor: Academia Brasilera de Música. 
y el oyente decodifica el mensaje, cada uno a su manera. Elegí la viola y el clarinete porque son instrumentos que transitan en una tesitura muy cercana, así como ocurre entre la "tesitura" del agua y la música. El piano funciona como el polo agregador de estos dos instrumentos.

No se presenta descripción de la partitura, pues no fue posible su consecución.

\section{Fantasia sobre "a muie rendêrá", Op. 1f. Liduino Pitombeira (Brasil) $^{8}$}

Escrita en 1988 y terminada en 2010. Esta obra fue adaptada a diversos formatos instrumentales, para completar así ocho combinaciones (Op. 1a, b, c, d, e, f, g y h), aunque la primera fue escrita para para clavicémbalo y viola da gamba, como parte de un montaje académico con el aquel entonces grupo musical del compositor.

Muié rendêra es una melodía famosa, posiblemente escrita por Virgulino Ferreira da Silva, también conocida como Lampião, una figura legendaria de la tradición del noreste de Brasil. La versión aportada por el compositor se encuentra escrita para clarinete en $\mathrm{Bb}$, violín (viola) y piano, en forma sonata, con cambios métricos que alternan compases irregulares con binarios. La melodía de la canción original es presentada por la viola y luego acompañada por el clarinete, siempre en contrapunto a la tercera, con algunas variaciones rítmicas y melódicas. El piano inicialmente acompaña la suave melodía con ritmo y armonía contrastantes, pero no imita ideas de la canción original; por el contrario, presenta con claridad los centros tonales propuestos y se une a los instrumentos melódicos para dar paso a las transiciones de cada sección, que desarrollan una idea musical antes de regresar al tema principal (A-Trio, 2015.).

\section{Figura 7. Partitura general (piano) con la melodía principal expuesta por la viola (fragmento)}

Score in $\mathrm{C}$ Fantasia sobre a Muié Rendêra

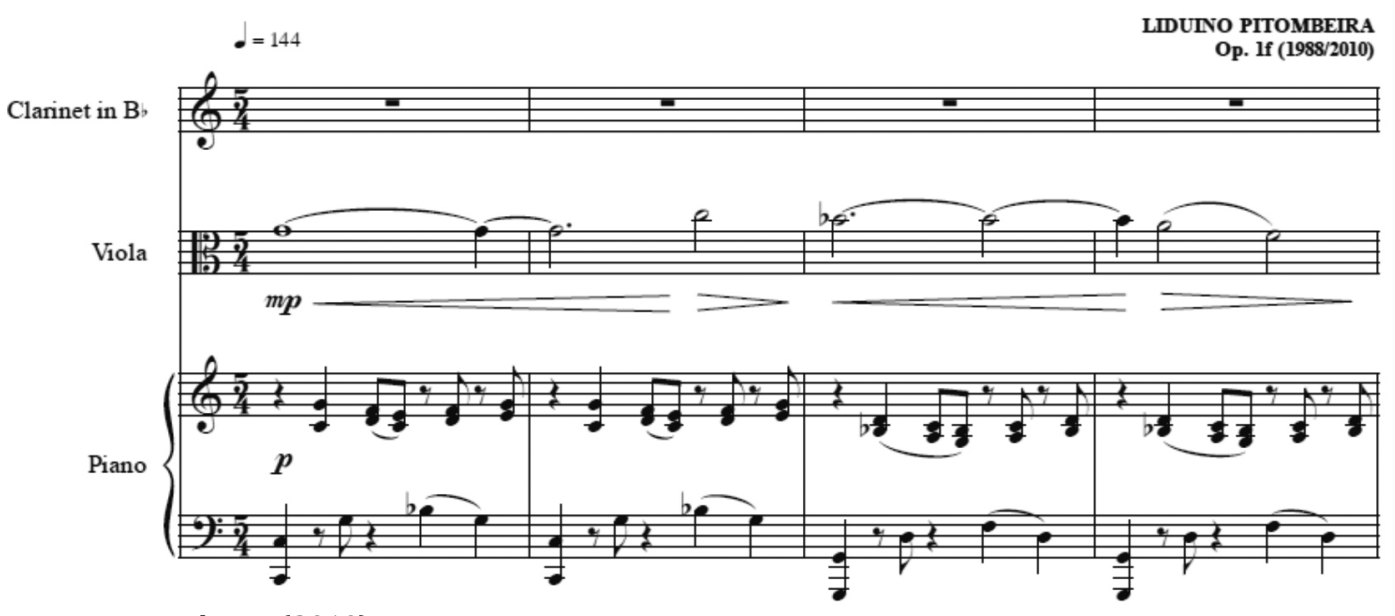

Fuente: Pitombeira (2010).

8 Partitura aportada por el autor. Consultar contacto en lista de referencias. 


\section{Brasiliana, 0p. 173c. Liduino Pitombeira (Brasil) ${ }^{9}$}

El compositor inició su escritura en 2011 y la terminó en 2016. Original para flauta, clarinete y piano por encargo del trio Brasilianas, compuesto por: Felicia Coelho (flauta), Aynara Silva (clarinete) y María Di Cavalcanti (piano). Sin embargo, el compositor ha escrito diversas versiones para varias de sus piezas y en este caso existe también una versión para violín, clarinete y piano. Estrenada por el trío Brasilianas en el Conservatorio Pernambucano de Música, en Recife, el día 8 de junio de 2011 (Trio Contrastes, 2016). Los títulos de los tres movimientos proceden de tres textos de Machado de Assis, que son:

\section{Flor anônima}

2. Ao acaso

3. O relógio de ouro

La obra emplea atonalismo libre; el primer movimiento es el más melódico de todos y presenta un juego musical entre los instrumentos melódicos y el piano, que emplea registros extremos hacia los bajos y crea tensión, no solo en este movimiento sino a lo largo de la obra. En el tercer movimiento suceden ideas musicales parecidas al primer movimiento, solo que las frases melódicas se proponen al unísono en el clarinete y la viola. El segundo movimiento, por el contrario, hace uso de notación gráfica y es necesario el empleo de técnicas extendidas en el piano y de registros extremos en clarinete y viola.

Figura 8. Partitura general (piano) correspondiente al segundo movimiento

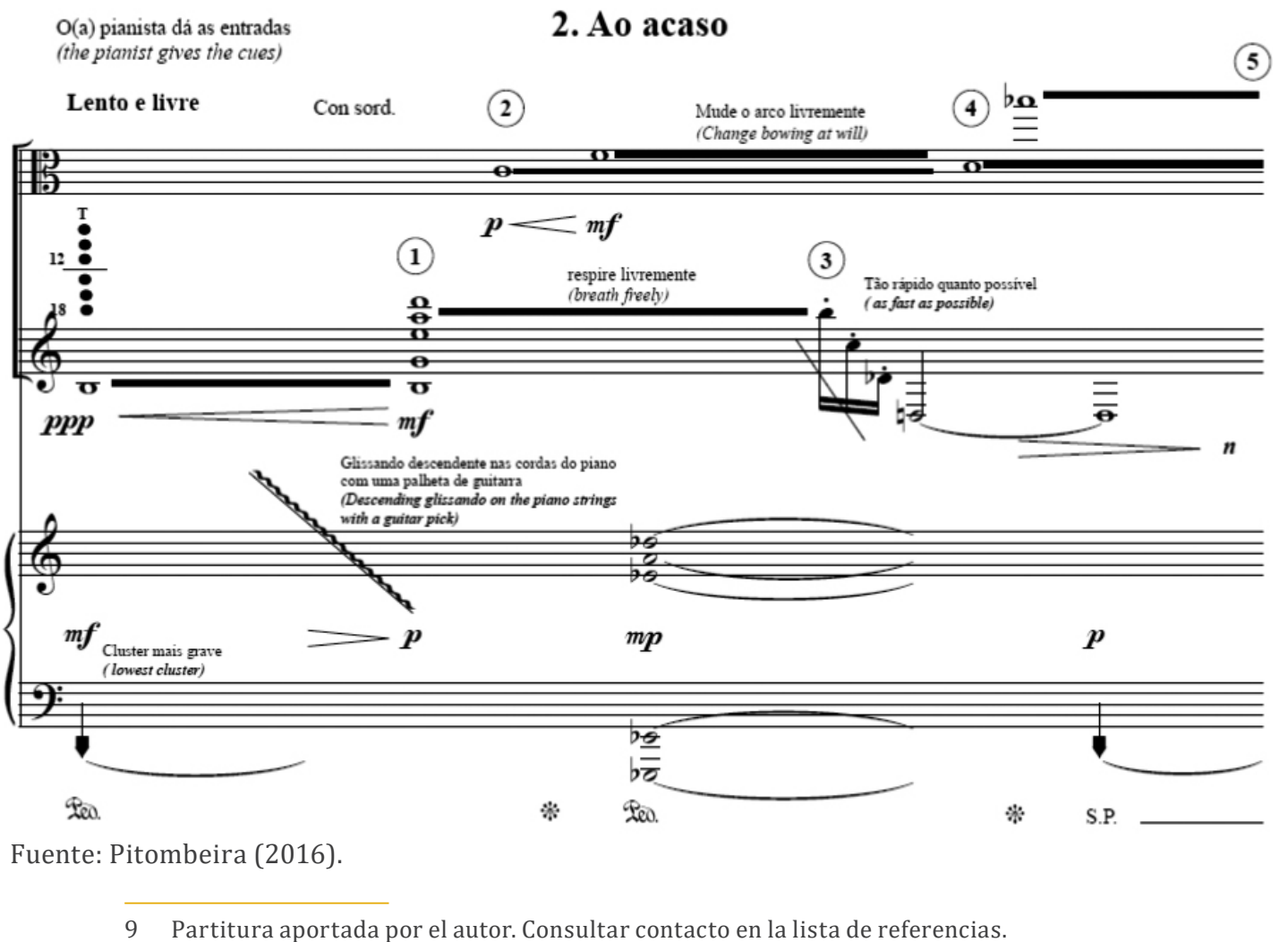




\section{Paineiras, 0p. 200. Liduino Pitombeira (Brasil) ${ }^{10}$}

Escrita en 2015, comisionada por el Trio Paineiras (Pitombeira, 2017), integrado por Marco Catto (violín/viola), José Batista J. (clarinete/clarinete bajo) y Marina Spoladore (piano9, quienes, por su versatilidad en varios instrumentos, sugirieron al compositor incluirlos en la obra, lo que dio como resultado la escritura de tres movimientos, distribuidos de la siguiente manera:

1. Lembrança em turbilhão: Para viola, clarinete bajo en $\mathrm{Bb}$ y piano

2. Saudades ao redor: para violín, clarinete en Bb y piano

3. Dispersos: para violín, clarinete en Bb y piano.

Los títulos de los movimientos se extrajeron del poema A paineira e o poente, de J. G. de Jorge Araújo (1914-1987). La obra emplea atonalismo libre y armonía cromática. El primer movimiento en realidad nos ilustra un torbellino, con mucho movimiento, inicialmente al unísono en los tres instrumentos y luego con un insistente ostinato del piano, mientras la viola y el clarinete, o bien llevan melodías al unísono o se alternan. La característica más palpable de este movimiento es la oscuridad sonora, que emplea registros medios a bajos en los tres instrumentos.

El segundo movimiento nos presenta una obra bastante melancólica, en la que se combinan de manera efectiva distintos registros de cada instrumento con frases más legato. En el tercer movimiento es interesante como "dispersa" los registros de los instrumentos para construir frases al unísono y, sin embargo, cada instrumento es diferenciable al oído. Contiene secciones muy rítmicas, lideradas por el piano, así como también un gran despliegue melódico, muy lírico, en el que cada instrumento tiene protagonismo.

\section{Figura 9. Fragmento inicial del tercer movimiento}

\section{Dispersos}

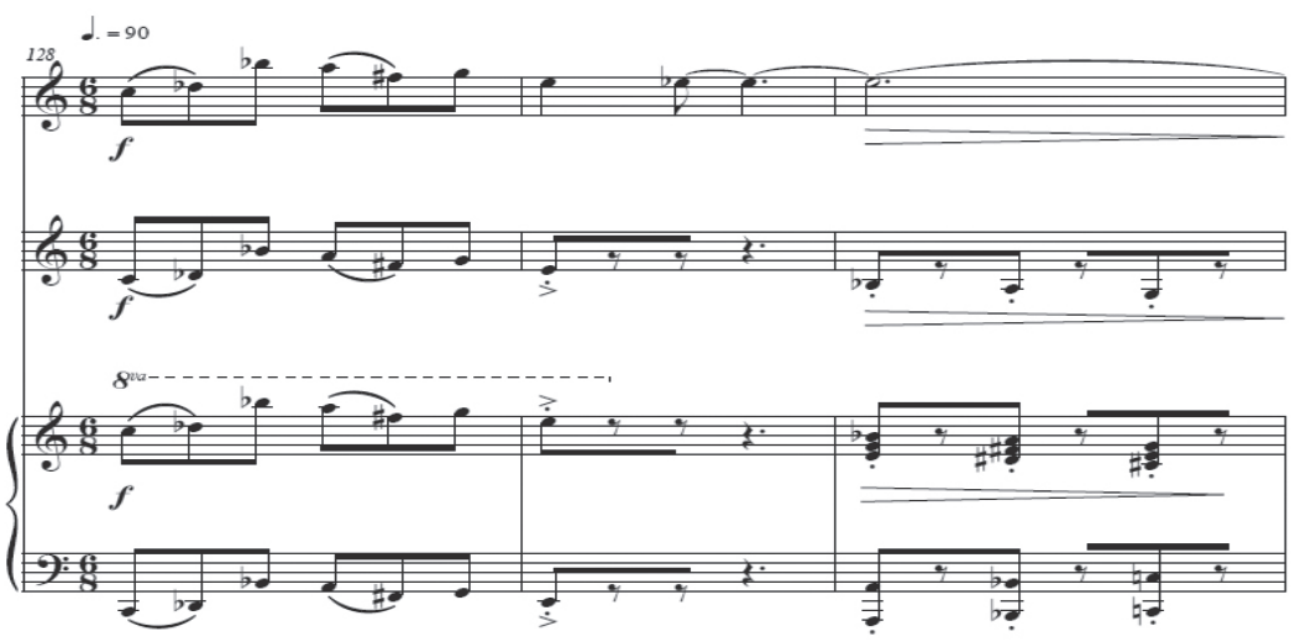

Fuente: Pitombeira (2015b).

10 Partitura aportada por el autor. Consultar contacto en la lista de referencias. 


\section{Mitología. José Revelo Burbano. (Colombia) ${ }^{11}$}

Obra escrita en 1998, originalmente para clarinete en Bb, bajo, bandola andina y guitarra. Adaptada para el formato de trío para viola, clarinete y piano por el mismo autor. Ganadora en el Encuentro Nacional de Tríos, Popayán (Colombia), en 1998. Se encuentra publicadas en el libro Arreglos para banda sinfónica, compositores nariñenses, de José Revelo Burbano, impreso por la Universidad de Nariño (Colombia) en 2009.

Escrita en ritmo de bambuco, en lenguaje tonal. El piano por lo general acompaña las melodías propuestas para el clarinete y la viola, que se acompañan en contrapunto o en contramelodías. Los instrumentistas no necesitan un gran despliegue técnico, sino un entendimiento de este aire popular colombiano.

Figura 10. Fragmento entre compases 12 y 17

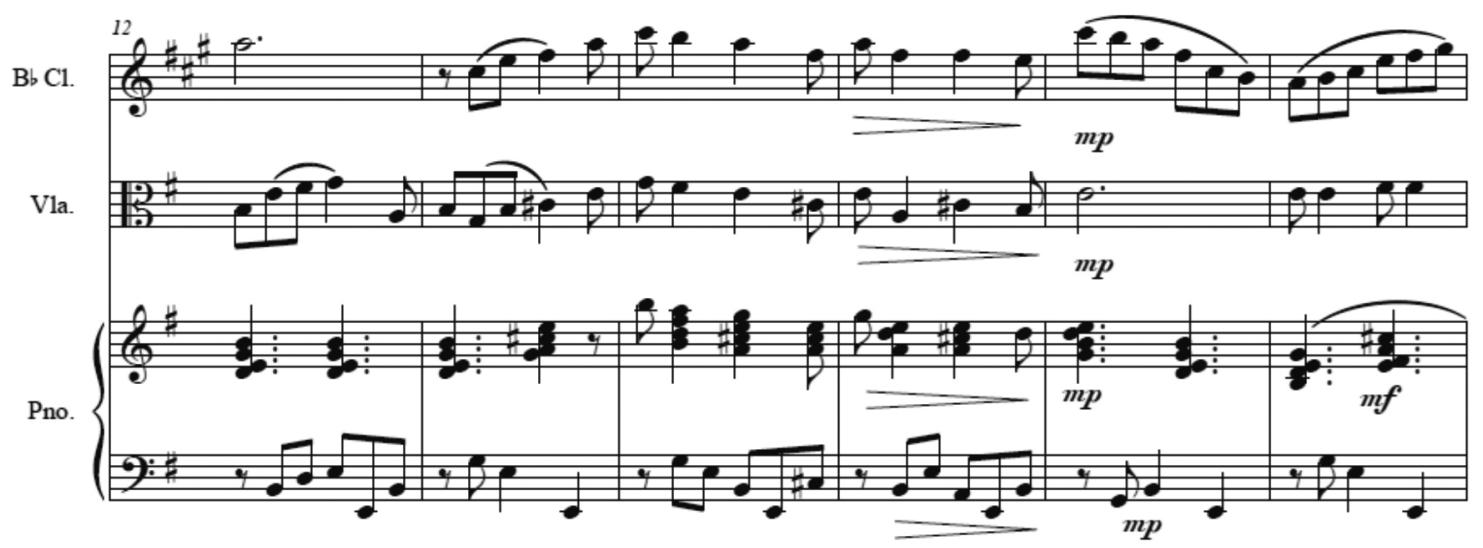

Fuente: Revelo Burbano (1998).

\section{Eco milenario. José Revelo Burbano (Colombia) ${ }^{12}$}

Obra escrita en 2002 y grabada por el Fondo Mixto de Cultura del Departamento de Nariño (Colombia) el mismo año. Originalmente escrita para guitarra, quena y charango. Adaptada para el formato de trío para viola, clarinete y piano por el autor.

Al igual que la anterior obra, se encuentra escrita en ritmo de bambuco. Sin embargo, aquí podemos notar armonías más complejas con agregaciones y dominantes, y más conversación entre los instrumentos. En algunos pasajes la mano izquierda propone melodías, mientras la derecha enfatiza el ritmo. Es claro el juego de pregunta y respuesta entre el piano y los instrumentos melódicos. No es necesario un gran despliegue técnico, pero sí un claro entendimiento de este aire popular colombiano.

11 Partitura aportada por el autor. Consultar contacto en la lista de referencias.

12 Partitura aportada por el autor. Consultar contacto en la lista de referencias. 
Figura 11. Fragmento desde el compás 41 hasta el 45

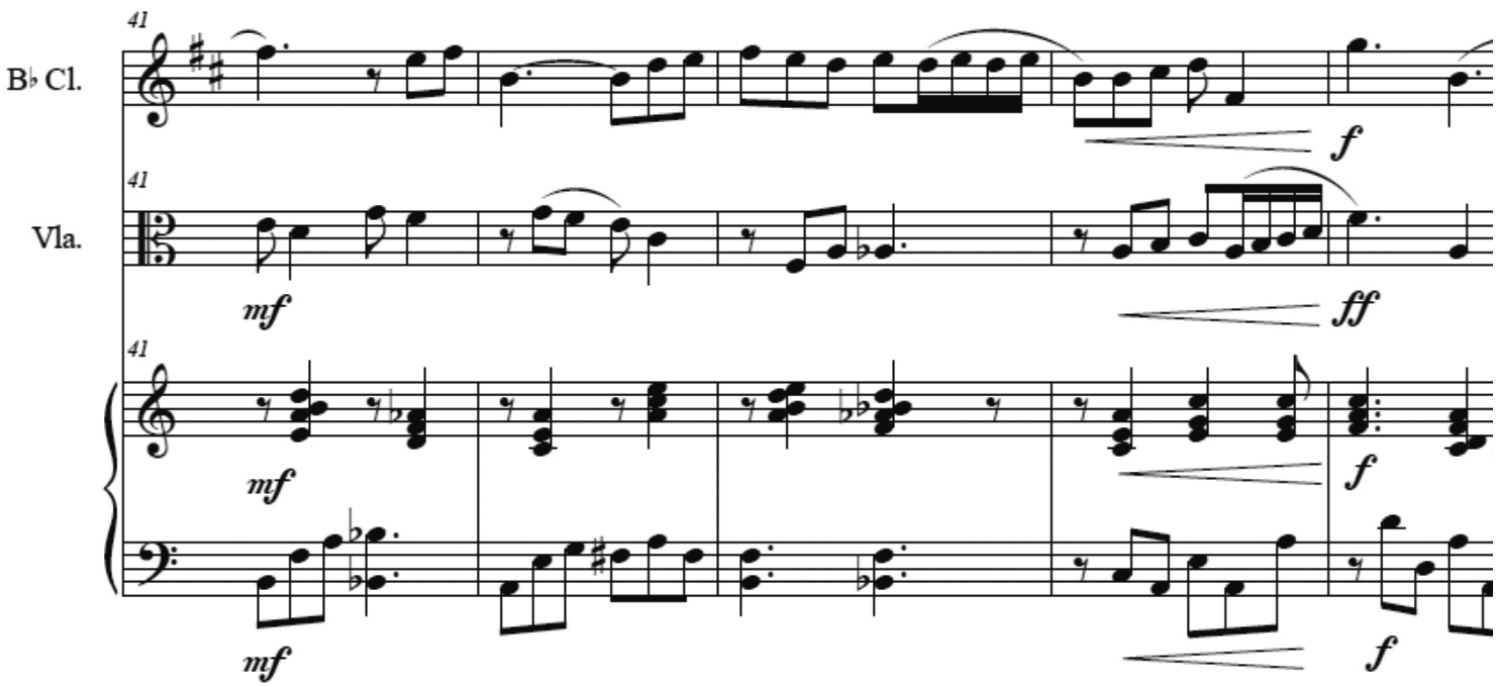

Fuente: Revelo Burbano (2002).

\section{Janus. Marco Alunno (Italia-Colombia)}

Esta obra fue originalmente escrita para flauta, viola y piano, pero el compositor la trascribió para clarinete, viola y piano a pedido del trío colombiano Acuarimántima (Acuarimántima Trío, 2017). La pieza presenta seis movimientos cortos, tres con una indicación de $\boldsymbol{d}=116$ y los otros tres de $\delta=72$, en las que se alteran no solo los tempi, sino también el carácter y la intensidad. El compositor da tres posibilidades para la realización de la obra. Las seis piezas cortas de Janus pueden tocarse en el siguiente orden:

- Directamente, como aparecen en el score, sin interrupción

- I-III-IV / II-IV-VI, con una pausa entre el primer grupo y el segundo

- II-IV-VI / I-III-IV, con una pausa entre el primer grupo y el segundo

(Alunno, s.f.).

Está escrita en un lenguaje aleatorio, con uso de técnicas extendidas en los movimientos I, III y V (rápidos), con indicaciones claras de duración de los sonidos, tempo y ejecución para el piano (manipulación de las cuerdas del piano para lograr efectos y cluster), viola (armónicos y sonidos indeterminados) y clarinete (efectos con la boquilla). 
Figura 12. Compases finales del primer movimiento

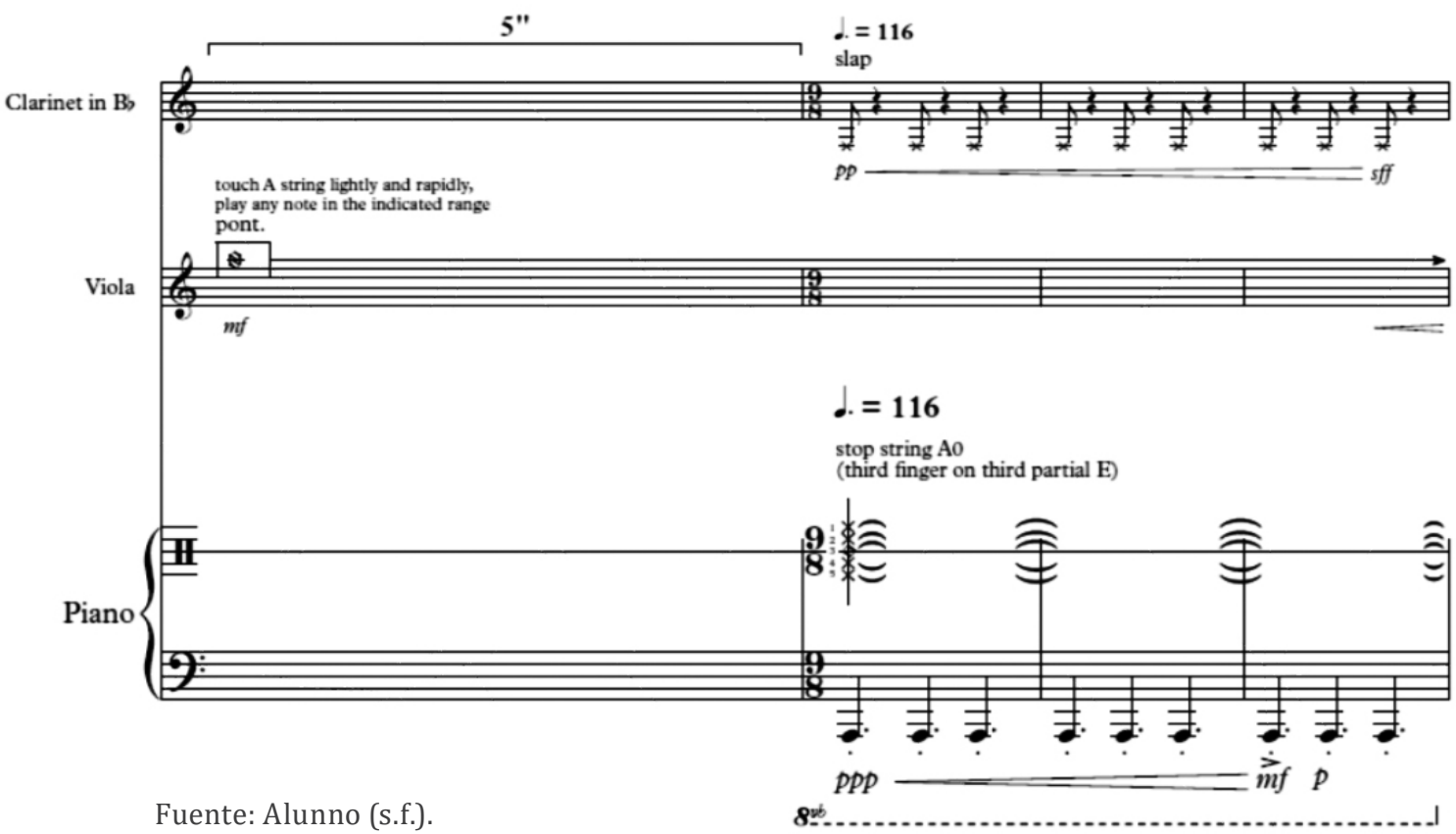

\section{La delgadina. Alejandro Cardona (Costa Rica) ${ }^{13}$}

Escrita en 1992 y revisada por el autor en 2001. Esta obra está basada en un canto popular costarricense conocido como La delgadina y otro popular venezolano, Canto de pilón. Escrita en atonalismo libre. El autor realiza una serie de variaciones mediante la intercalación de motivos de cada uno de los cantos, repartidos en nocturnos:

I. La delgadina (nocturno primero), íntimo $\delta:=126$.

En el primer movimiento, el clarinete y la viola nos presentan notas pedales en registros medios, mientras que el piano realiza pequeños motivos, muy rítmicos, por medio de registros extremos desde sol\# 1 hasta fa 7, que se hace densa poco a poco, hasta llegar a que la viola imite los motivos del piano (compás 50).

II. Canto de pilón 1, rítmico d. $=76$.

Movimiento más rítmico y contrapuntístico. El piano mantiene protagonismo con rítmicas más complejas y mediante registros extremos. El clarinete tiene mucha más melodía, acompañada por pequeñas células rítmicas en la viola. Presenta gran variedad de dinámicas en cada instrumento, lo que crea un juego sonoro denso.

III. La delgadina (Nocturno segundo), $d=$ íntimo $=d 6$.

Este movimiento contrasta con el anterior y repite material melódico y rítmico usado en el primer nocturno. La variación se encuentra en algunos motivos melódicos del

13. Partitura aportada por el autor. Consultar contacto en la lista de referencias. 
clarinete, así como uso de armónicos en la viola, con los que se pretende, tal vez, cambiar la atmósfera de la pieza, a pesar de su parecido con el primer nocturno.

IV. Canto de pilón $2 . d=d$., rítmico $d=76$.

De nuevo el autor nos presenta un movimiento más rítmico; sin embargo, en esta sección el piano cumple una función más rítmica y el clarinete y la viola presentan material melódico más rico y denso. Hacia la segunda casilla hasta el final (compás 205) utiliza ideas ya expuestas en los cantos de pilón: notas pedales en clarinete y viola y versatilidad motívica en el piano.

\section{La delgadina (tonada), cantabile $d=48$.}

La última sección de la obra propone una expresividad mayor, iniciada por el piano y seguida por motivos melódicos del clarinete en registro medio y agudo, mientras que la viola es la que lleva la densidad rítmica, con saltos interválicos de segundas hasta trecenas, con mucha variedad dinámica, con lo que adquiere protagonismo durante toda la sección. El movimiento finaliza con ideas usadas en los nocturnos: notas pedales en instrumentos melódicos y motivos rítmicos con registros extremos en el piano.

\section{Figura 13. Fragmento del segundo movimiento. Densidad rítmica y melódica}

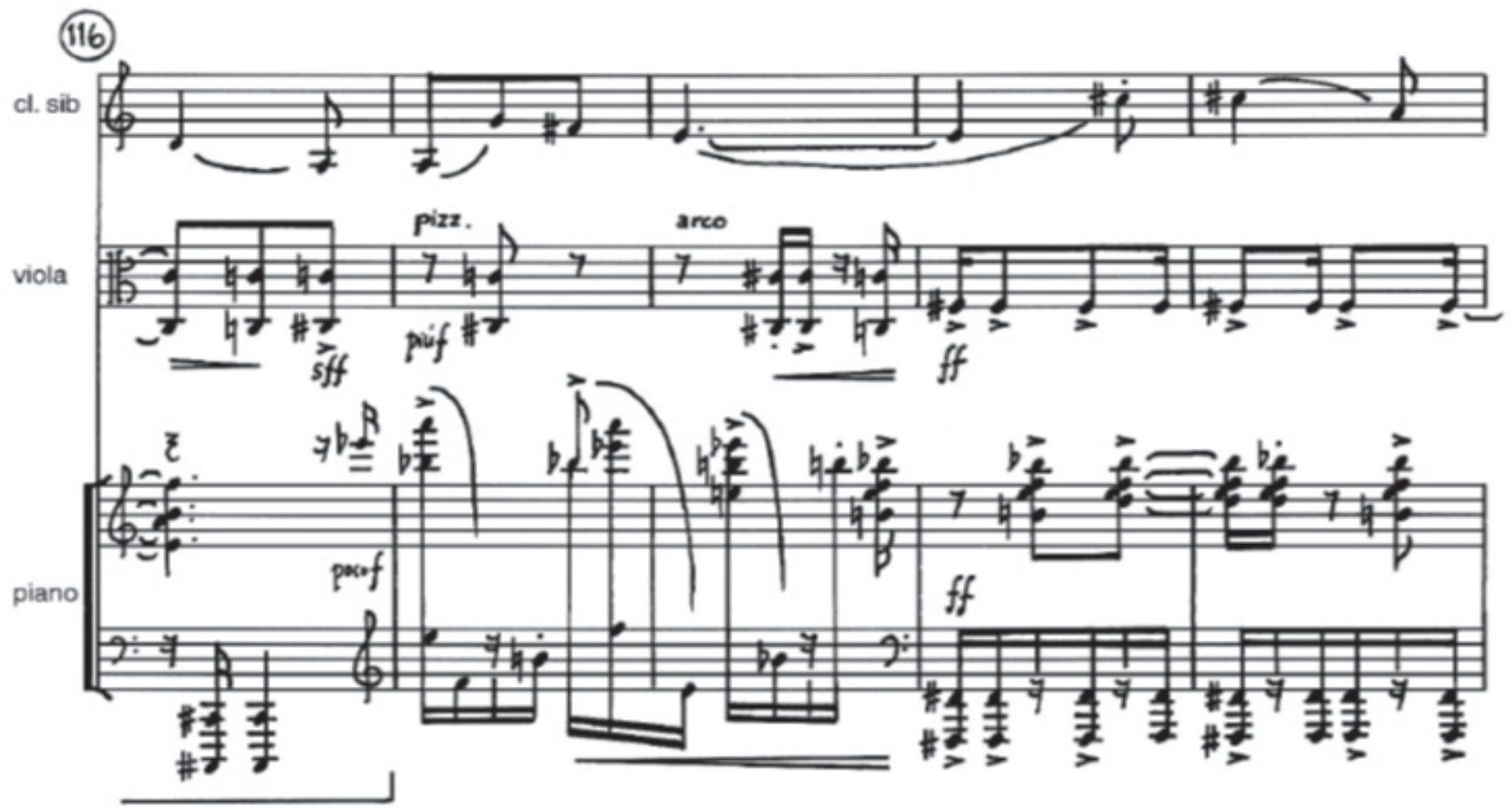

Fuente: Cardona (2001). 


\section{El loco y la emperatriz en la recámara de los espejos. Carlos José Castro Mora (Costa Rica) ${ }^{14}$}

Obra comisionada por la organización "Una hora de música", que presentaba conciertos de música de cámara en el Teatro Nacional de Costa Rica. Fue estrenada en 1989 por los maestros Yamileth Pérez en el clarinete, Luis Carlos Amador en la viola y Gertrudis Feterman en el piano.

Escrita para clarinete en la. Esta obra presenta con claridad una escena de persecución, muy nítida, por la velocidad y por las métricas irregulares que utiliza en alternancia al 6/8 inicial (7/8 y 5/8); incluso, usa la métrica 13/8 luego de una secuencia métrica: 6/8-8/8-12/8. Si la densidad no está dada por la rítmica usada, sí lo está en la densidad sonora de las escalas usadas o en la superposición de melodías entre los tres instrumentos.

Figura 14. Fragmento de la parte general (piano). Evidencia los cambios métricos sucesivos

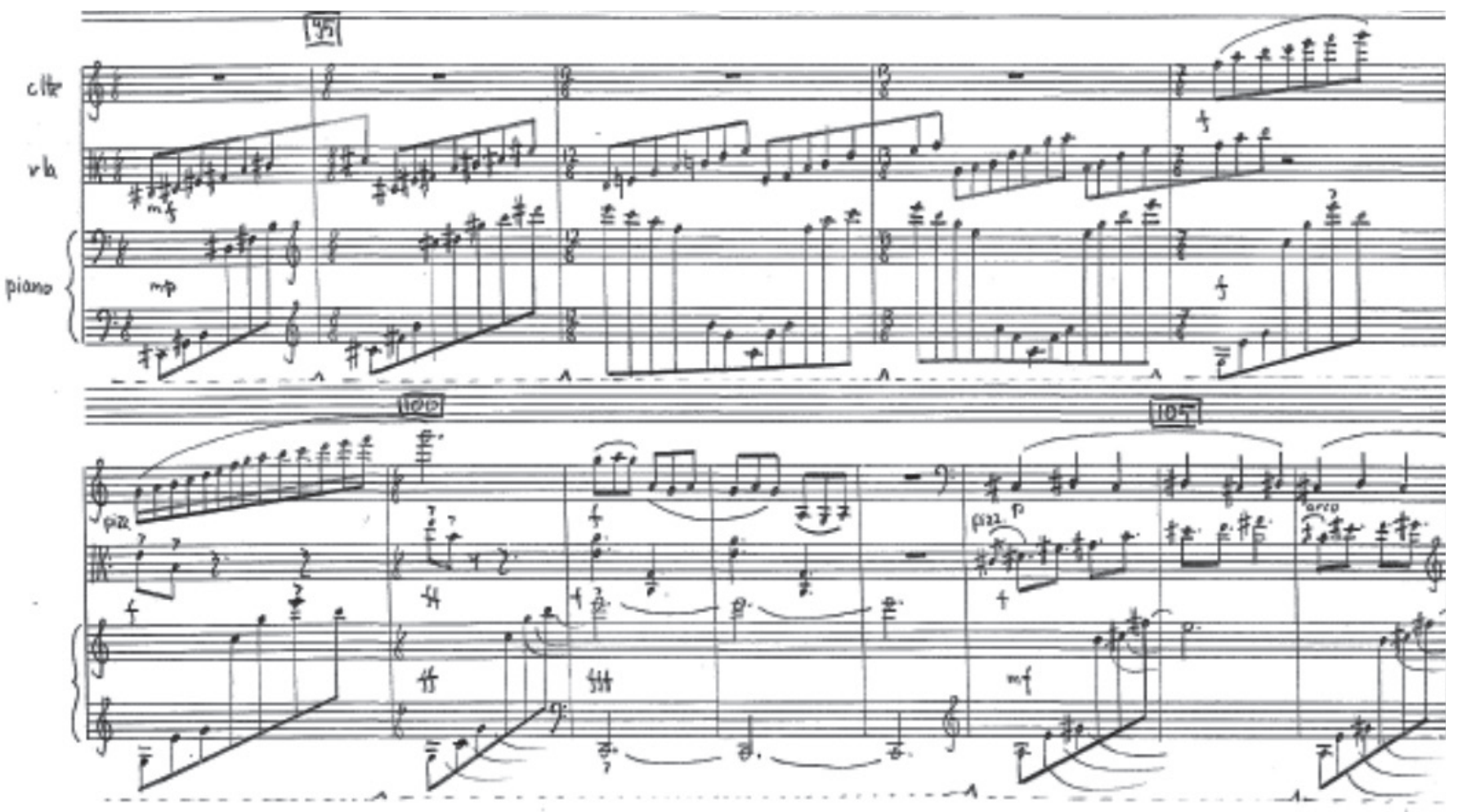

Fuente: Castro Mora (1989).

14 Partitura aportada por el autor. Consultar contacto en la lista de referencias. 


\section{Trío $N^{\circ}$ 1. Adrián Ortiz Rivera (Costa Rica) ${ }^{15}$}

Obra escrita en 1994, compuesta por iniciativa del autor como parte de las obras de su recital de composición para licenciatura, y fue estrenada en 1995. En palabras del autor: "Me pareció una interesante combinación de instrumentos. Ha sido interpretada en varias ocasiones en distintos lugares, pero aún no existe grabación" (Ortiz Rivera, en entrevista concedida por correo electrónico a la autora del artículo el 23 de octubre de 2017).

Escrita en lenguaje modal, muy contrapuntística entre los instrumentos melódicos y en tres movimientos sin título o aire interpretativo específico: I. $d=65$, II. d=96 y III. $d=79$. El primer movimiento presenta un acompañamiento del piano a las melodías propuestas por el clarinete y la viola de manera alternada. El segundo movimiento, con cambios métricos constantes, en los que predominan figuraciones ternarias (tresillos), que crean un juego rítmico y melódico entre los tres instrumentos. El tercer movimiento presenta, también, cambios métricos constantes, pero ahora irregulares; el piano tiene mayor presencia melódica, en juego con los motivos de la viola y el clarinete.

\section{Figura 15. Fragmento de la parte de piano el segundo movimiento. Presenta juego rítmico ternario que predomina en esta sección}

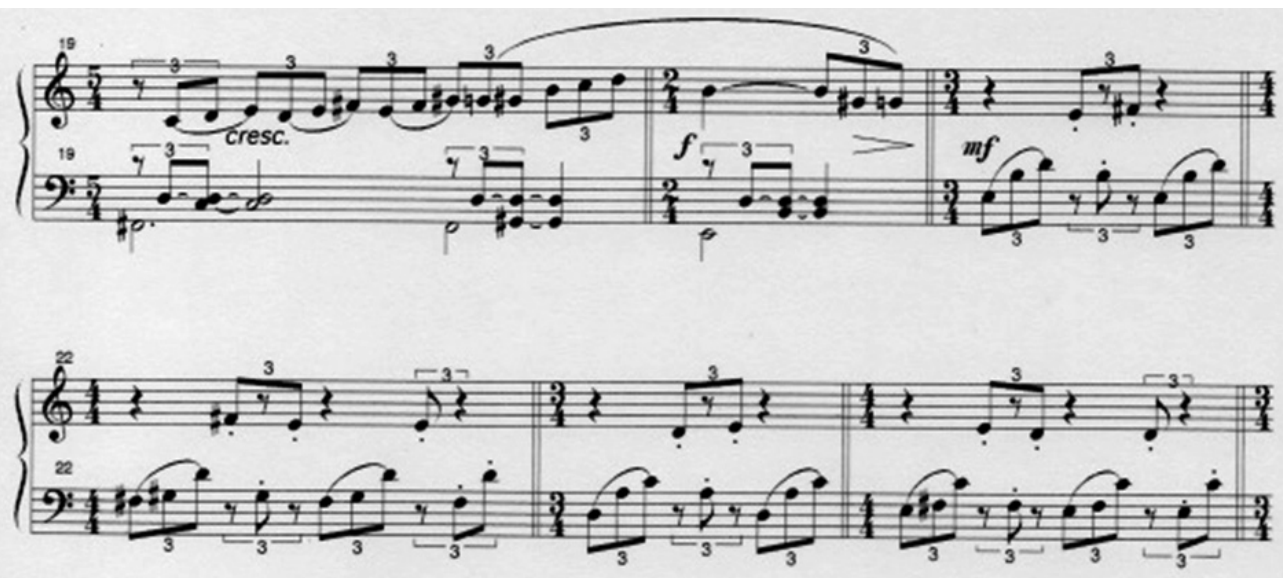

Fuente: Ortiz Rivera (1994).

\section{Música concertante. Sergio Delgado (Costa Rica) ${ }^{16}$}

Obra escrita entre junio y septiembre del año 2016. Originalmente fue una solicitud de la Dra. Yamileth Pérez, profesora catedrática de clarinete de la Universidad de Costa Rica; sin embargo, no fue estrenada. Estaba proyectada para ser ejecutada por

15 Partitura aportada por el autor. Consultar contacto en la lista de referencias.

16 Partitura aportada por el autor. Consultar contacto en la lista de referencias. 
maestros del Departamento de Música la Universidad de Costa Rica; no obstante, los diversos compromisos de la agrupación impidieron llevarla a cabo.

Escrita para clarinete en la. En su mayor parte está escrita en 6/8, con el fin de evocar "el tambito", ritmo folclórico costarricense. Está articulada en cuatro secciones muy claras, aunque interpretadas de manera ininterrumpida:

I. Moderato e giocoso d. = c. 62 , en la que se señala una métrica de 6/8. Es recurrente el efecto de viola col legno, con marcados cambios de tempo como meno mosso hacia el compás 87, accelerando en el 96 para regresar al tempo original en el compás 98.

II. Largo $\oint^{\prime}=72$ (hacia compás 128)

III. Scherzando ل $=72$ (en compás 173). En esta sección realizan un dúo el clarinete y el piano. Están demarcados cambios de tempo como: $3 / 8,2 / 4(\delta=\delta)$

IV. Presto. Sección más corta, con 20 compases, pero que anuncian el final de la pieza.

\section{Figura 16. Solo de piano. Similitud del ritmo costarricense "tambito" con el pasillo colombiano, motivo recurrente en la obra}
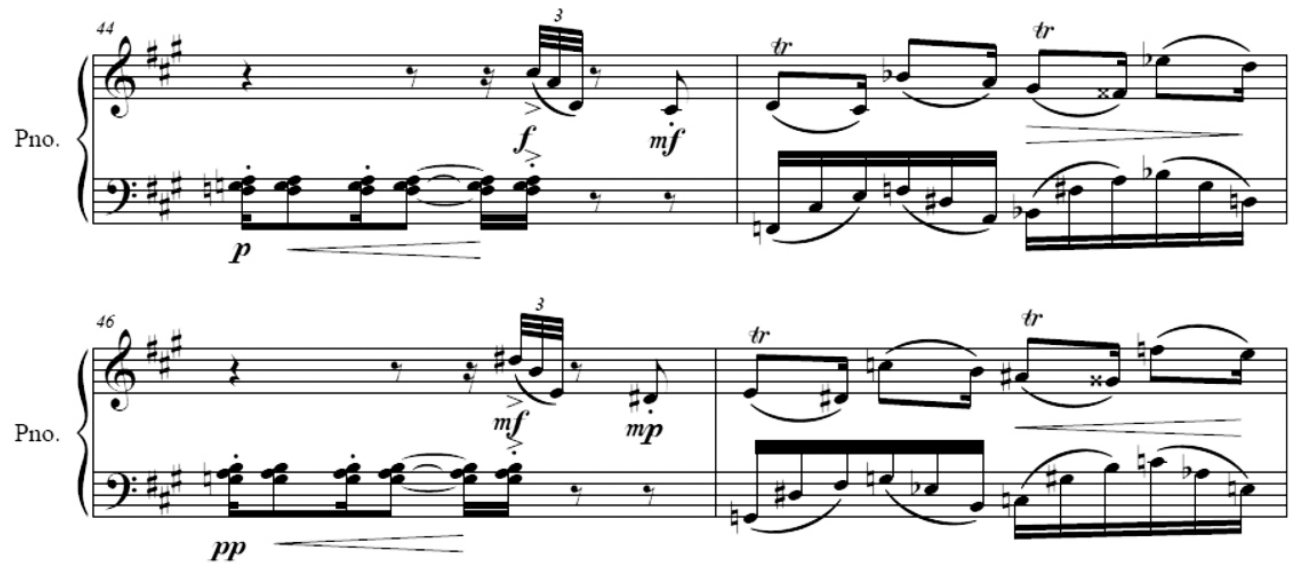

Fuente: Delgado (2016).

\section{Sweet CeciMar. Jorge López Marín (Cuba) ${ }^{17}$}

Originalmente escrita para flauta, clarinete y piano en 2002. Existen transcripciones para dos saxofones, clarinete, flauta y piano, lo mismo que para violín y piano, publicadas por la editorial Gebr. Stark Leipzig. La versión para trío de clarinete, viola y piano se realizó para que fuese grabada en el disco compacto "Jorge López", del sello discográfico Colibrí Records. La grabación estuvo a cargo del trío D’Acord. La obra contiene cuatro partes y fue estrenada por Marta Salgado (viola), Vicente Monterrey (clarinete) y Marita Rodríguez (piano).

17 No fue posible la consecución de la obra por asuntos de edición. Consultar contacto en la lista de referencias. 


\section{Trío Claros, Op. 77. Marlon Herrera (Honduras) ${ }^{18}$}

Obra escrita en 2018, con dedicatoria a Franklin F. F Claros. Para clarinete en Bb $^{19}$, escrita en tres movimientos. Predomina melodía con acompañamiento, con algunos motivos contrapuntísticos entre la viola y el clarinete.

Primer movimiento: andante $d=80$. Obra rica desde el punto de vista rítmico, en la que la viola adquiere protagonismo, mientras que el clarinete acompaña con motivos legatto y el piano mantiene un movimiento activo y constante, con ostinato sobre la misma armonía. El segundo movimiento, lento $\mathcal{\delta}=120$. Contiene cambios métricos irregulares constantes, en los que el piano mantiene su función acompañante y rítmicamente estable, mientras que la viola presenta el material melódico de la pieza. En la entrada del clarinete en registro bajo, la viola inicia su papel acompañante en el que dobla al piano. Hacia el compás 40, la viola y el clarinete desarrollan una melodía por distancia de cuartas, mientras que el piano despliega melismas que varían cada dos compases. Finaliza la viola con la reexposición de la melodía inicial, mientras que el piano hace acordes con agregaciones y clusters de manera arpegiada.

El último movimiento, allegro $d$ : 90, nos presenta un movimiento al unísono de los tres instrumentos por movimiento cromático, por medio de la exploración de los registros extremos de cada instrumento. Hacia el compás 12 se presenta una variación rítmica de clusters en el piano y ostinato en la viola, mientras que el clarinete ejecuta una melodía poco cambiante en corcheas y movimiento contrario a la viola.

\section{Figura 17. Fragmento del primer movimiento, primeros compases}

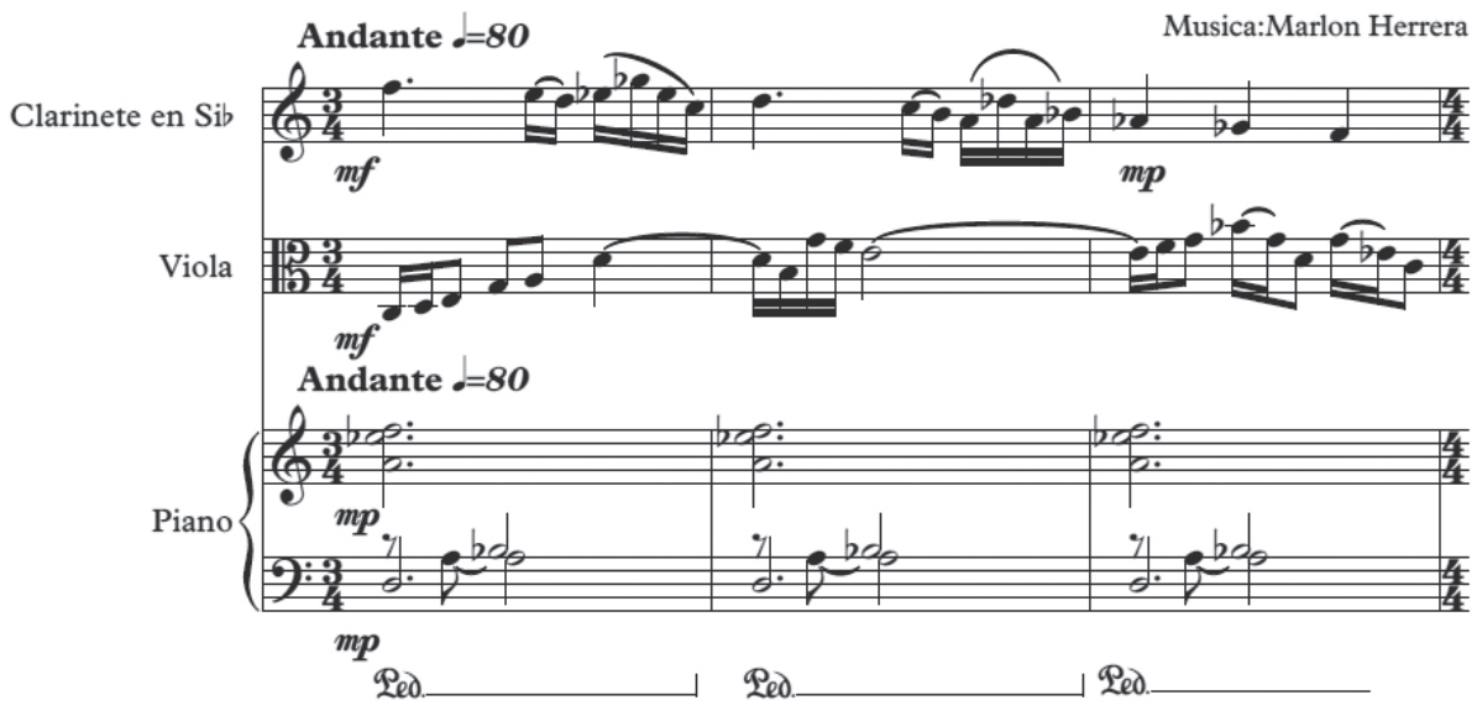

Fuente: Gebr. Stark Musicverlag (2017).

18 Partitura aportada por el autor. Consultar contacto en la lista de referencias.

19 Partitura del clarinete en do. 


\section{Ofrenda. Leonardo Coral (México) ${ }^{20}$}

Escrita en 2003. La obra Piezas fantásticas, de Leonardo Coral, para violín y piano y que contiene cinco movimientos, es la composición de la que se origina esta pieza. Compuesta en un solo movimiento, con indicación Expresivo, que toma ideas musicales del primer movimiento, Preludio, y de ideas extramusicales del segundo, La muerte danzando.

Para esta versión, comisionada por el trío compuesto por Ismael Sánchez (clarinete), Matthew Schubring (viola) y María Teresa Frenk (piano), el compositor desarrolló el plan temático de la versión original; de igual manera utilizó el color de los instrumentos para crear una obra distinguible con facilidad de la primera, en la que combinó los registros medios de los instrumentos con el fin de crear una mezcla más compacta y homogénea. Las melodías propuestas son bastantes líricas y evocan a Debussy. Encontramos también muchos cambios métricos, que le dan mucho movimiento a la obra en compás de 5/4 sobre todo, que alterna con los compases en 3/4 o 4/4 en los que se generan puntos de encuentro (cadenciales) entre dos o los tres instrumentos (Acuarimántima, 2017).

\section{Figura 18. Primeros compases de la obra. Presenta la melodía inicial del preludio de las piezas fantásticas para violín y piano}

\section{Ofrenda}

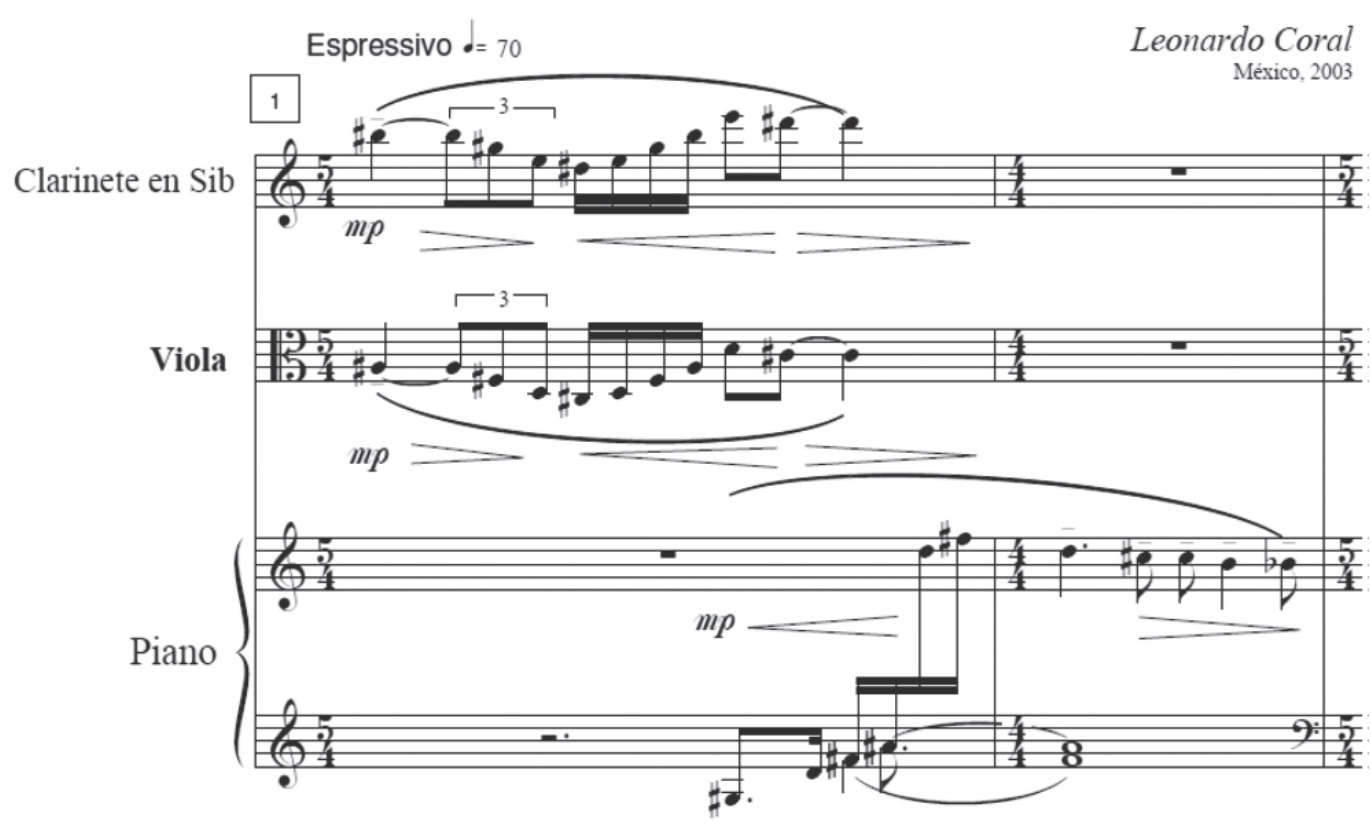

Fuente: Coral (2003).

20 Partitura aportada por el autor. Consultar contacto en la lista de referencias. 


\section{Circus. Ramón Romo Lizárraga (México)}

Fue escrita como parte de un proyecto universitario del cual en su interpretación fue participe Mario Lavista. No se describió esta obra en el presente artículo por encontrase sujeta a derechos de autor no cedidos por el compositor.

\section{LVTCN. Dan Román. (Puerto Rico) ${ }^{21}$}

Obra escrita originalmente para oboe, viola y piano; sin embargo, el autor, autorizó la transcripción del oboe a clarinete en Bb para la inclusión de su obra en esta referenciación de repertorio de cámara. Escrita para el Ensamble Schumann.

LVTCN (se puede pronunciar como Levitación) se compone de cinco movimientos cortos:

I. La montaña, luchando (d. = ca 120, 1:30 aproximadamente). Es un movimiento muy rítmico, siempre con denominador en 8 , con cambios de doce, seis, ocho, cinco, cuatro y dos pulsos de corchea por secciones de compases, siempre con marcaciones definidas en conjunto por los tres instrumentos. Hay clusters indicados para el piano con variaciones de registro y cromatismos sugeridos en la mano izquierda. La viola y el clarinete se comportan isorrítmicamente con él. De igual manera, hay momentos de quietud indicados, como Tacet, con advertencia de cambios de tempo.

II. Electro-stratro ( $d=$ ca $60,1: 30$ aproximadamente). Escrita sin mensuración de compás. El tempo se especifica por medición en segundos en cada sección. El ritmo indicado es el mismo para cada instrumento en las primeras cuatro secciones y con los mismos efectos. El piano siempre interactúa con los demás instrumentos con trémolo en alturas determinadas.

III. Color mostaza ( $d=94,1: 30$ aproximadamente). Esta sección, bastante rítmica como la primera, con variaciones de pulso siempre con denominador 4, pero con cambios de la acentuación con amalgamas de cuatro, dos, uno y tres pulsos de negra por compás. Se mantienen los sonidos agudos en los tres instrumentos en un rango de do 4 al si bemol 3, con excepción de la sección final, en la que se amplían los rangos iniciando por el piano, que acude a una octava más abajo del do 2. Es una sección muy densa, dada por la dinámica sugerida en casi todo el movimiento para los tres instrumentos: fff, además de los acentos en combinación con $s f z$ y en contraste con los registros agudos de cada instrumento.

IV. Lluvia al revés ( $d=110,1: 30$ aproximadamente). Este movimiento está propuesto con cambios métricos bastante seguidos en relación con la negra: 5/4, 3/4, 2/4 y 4/4; sin embargo, la propuesta del autor sobre la medida del tiempo está en relaciones de 4:5 en el caso del compás de 5/4, de 4:3 en el caso del compás de $3 / 4$ y así continua la secuencia, con excepción de los compases de 4/4, que se concierten en puntos de estabilización rítmica.

21 Partitura aportada por el autor. Consultar contacto en la lista de referencias. 
IV. Recuérdame (d = ca 50, 1:30 aproximadamente). Es el movimiento más lento de la obra. No tiene mensuración de compás; sin embargo, el tempo está medido en segundos. En los tres instrumentos utiliza escalas modales combinadas o en forma de canon.

Figura 19. Fragmento del primer movimiento. Presenta cambios rítmicos constantes

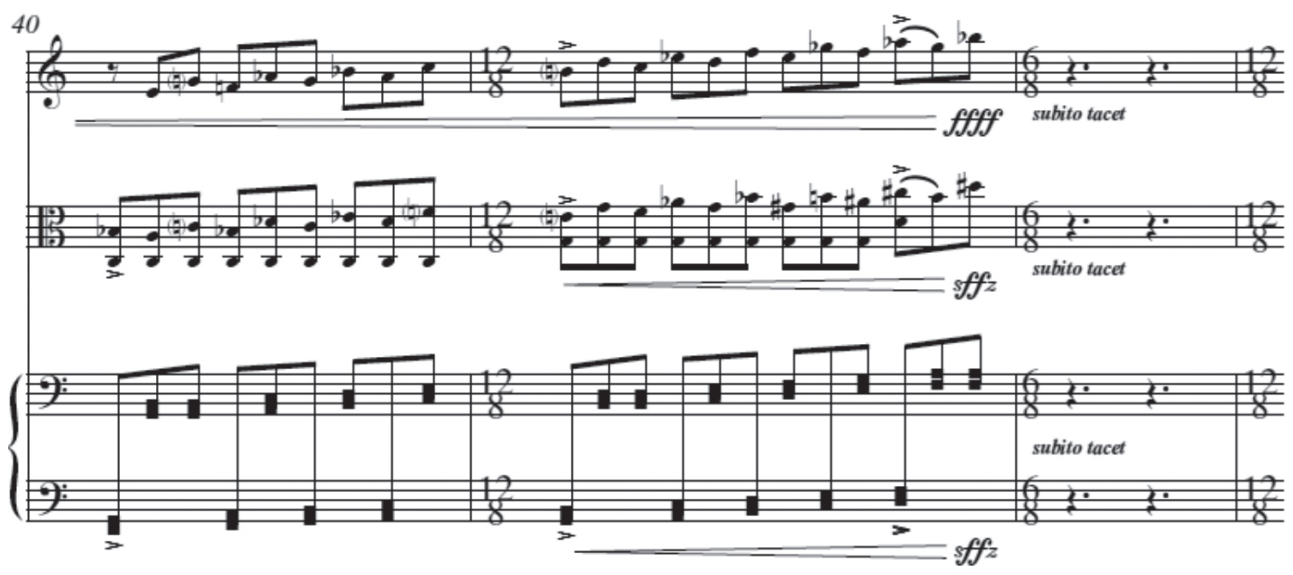

Fuente: Román (2016).

\section{Cuatro sombras de una palma. Carlos Alberto Vázquez (Puerto Rico)}

La obra fue compuesta en 2007, por encargo de un trío originario de Texas, Estados Unidos, aunque nunca fue interpretada por dicho grupo. Sin embargo, en 2015 fue grabada por músicos de la Orquesta Sinfónica de Puerto Rico y en 2017 por el trío colombiano Acuarimántima (Acuarimántima, 2017).

Escrito en cuatro movimientos, que se basan en cuatro obras literarias de escritores latinoamericanos reconocidos:

I. Makak's dream. Primer movimiento. Basado en el libro Dream on Monkey Mountain, de Derek Walcott. Escrita en lenguaje modal. Inicia un solo del piano y luego la viola y el clarinete hacen su entrada con un movimiento contrapuntístico. Hacia el compás 8 lleva al piano y al clarinete a registros extremos, con lo que se alcanza un punto de tensión que logra ser neutralizado por un movimiento descendente del piano y la entrada de la viola con armónicos naturales sobre la nota fa. Cambios constantes de métricas de irregulares (5/4) a regulares.

II. La desimprovisación de los Morrison. Segundo movimiento. Basado en la obra Quíntuples, del dramaturgo Luis Rafael Sánchez. Indicación: caotico d =60. Métrica: 6/8. Por medio de la gran cantidad de efectos tensos usados, como sul ponticello en la viola, armónicos en registros extremos de la viola, contrapunto 
cromático en el piano e inclusión de acordes de tonos enteros, pasajes agudos con figuraciones ternarias en el clarinete y algunos glisandi bastante extensos para la viola y el clarinete en duraciones extensas, que denotan el caos y la tensión que describe la obra de teatro, en cuanto a la situación familiar de los Morrison.

III. Aquel aroma a jazmines muertos...Tercer movimiento, basado en la obra La hojarasca, de Gabriel García Márquez. El movimiento empieza con una serie de efectos especiales (técnicas extendidas) en los instrumentos: col legno en la viola, golpeteo de llaves en el clarinete y cuerdas tañidas en el piano con un plectro. El movimiento concluye con efectos similares a los del principio.

IV. La guayabera izada. Cuarto movimiento, basado en el cuento Encancaranublado, de Ana Lydia Vega. El piano lleva el hilo conductor de la historia, mediante la demarcación de los episodios cambiantes y los momentos clave del cuento, dada la mayor carga rítmica y armónica asignada, mientras la viola y el clarinete se acompañan isorrítmicamente, como sucede en los compases del 6 al 14, por ejemplo, o las ideas motívicas las desarrolla el clarinete por medio del adorno rítmico y melódico de las frases que lleva con la viola.

Figura 20. Fragmento de la parte de piano de Aquel aroma a jazmines muertos... en el que especifica los efectos necesarios para el piano

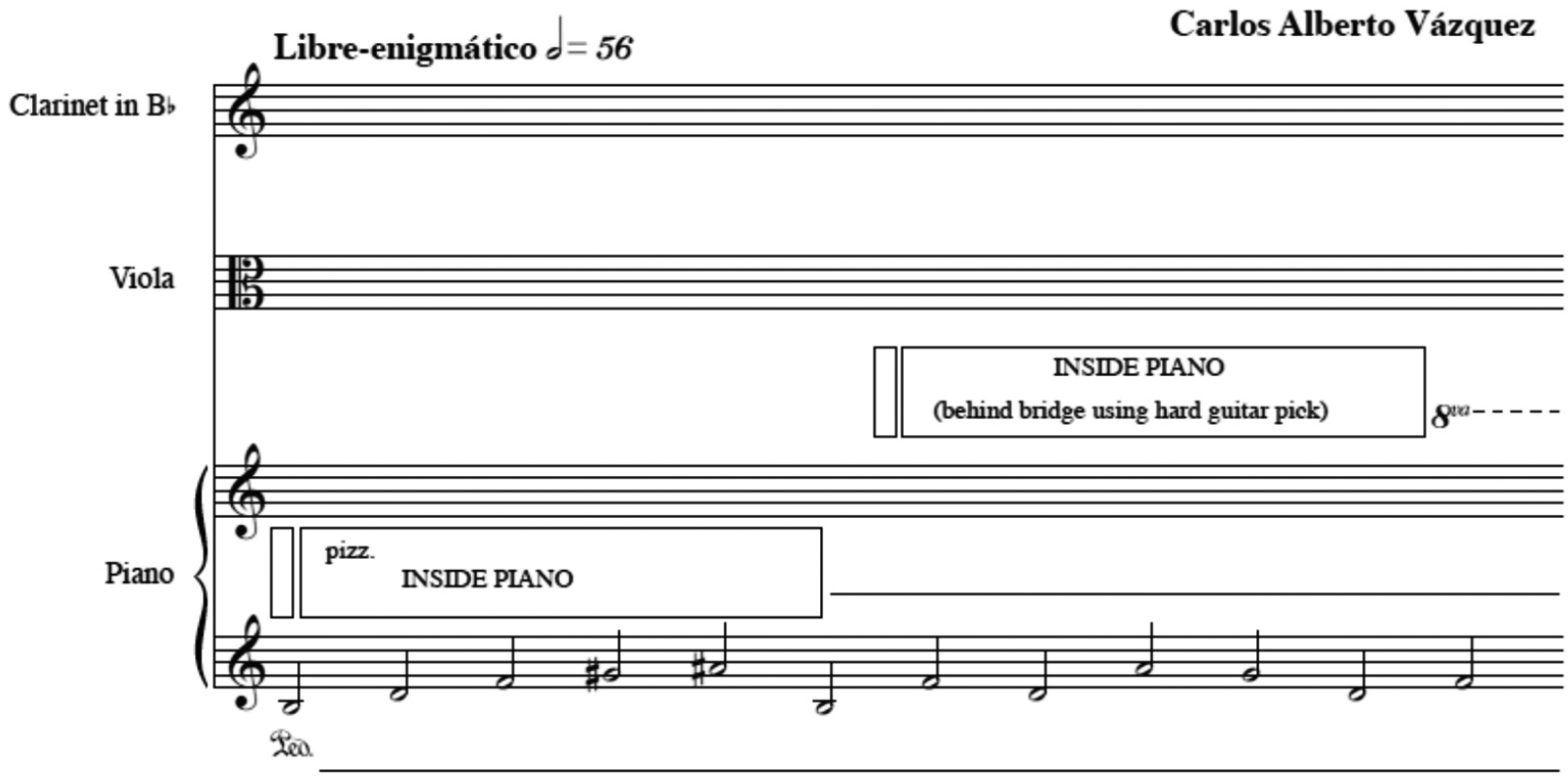

Fuente: Vázquez (2007). 


\section{Conclusiones}

La consecución de obras ha sido satisfactoria en cuanto a la respuesta de los compositores entrevistados, que respondieron que el formato propuesto es interesante y es una buena mezcla sonora. La mayoría de obras han sido adaptaciones comisionadas por los mismos intérpretes, pero que, sin duda, nos dejan un legado y mayores posibilidades musicales y estéticas en la música de cámara.

Las políticas de derechos de autor o, en algunos casos, las limitaciones de comunicación con algunos compositores no permitieron la disponibilidad de algunas partituras y por ello no hay mayores comentarios o descripción específica sobre las correspondientes obras. Es importante aprovechar todas las fuentes de comunicación posible, por lo que también es de resaltar que las redes sociales fueron un apoyo total en la ubicación de muchos compositores. Facebook-Messenger ayudó de manera significativa en el contacto y la posterior consulta de sitios web oficiales y de intercambio de experiencias con colegas instrumentistas, lo mismo que con muestras del trabajo grabado en audio o en video, como fuente referencial para la investigación.

El lenguaje en el que están escritas las obras, aunque no es complejo para nuestro tiempo, si es un poco ajeno a las costumbres de muchos instrumentistas. Se espera que las descripciones dadas en el presente escrito conduzcan a que el repertorio sea más llamativo para el formato instrumental estudiado y sean más conocidas a medida que sean publicadas y discutidas las consideraciones para tener en cuenta para la interpretación de este repertorio, con el fin de contar, además, con la oportunidad de poner en práctica las sugerencias de los compositores en vida, para el entendimiento de las obras a la hora de estudiarlas e interpretarlas.

Es importante reflexionar sobre la implementación del conocimiento de las obras nuevas en el ámbito académico y cómo los estudiantes a partir de pregrado perciben la música latinoamericana (Nobre, 1978), pues, entre los documentos encontrados como antecedentes de esta investigación, la mayoría de intérpretes se encuentran en nivel de doctorado (Montano, 2016; Stephenson, 2011; Vdóvina Vdóvina, 2006; Víquez Còrdoba, 2011), pero, si revisamos con atención la complejidad de algunas obras, pueden ser tenidas en cuenta en ensambles de menor nivel técnico, porque brindan esta posibilidad; es el caso de Janus, de Marco Alunno, Trío $N^{\circ} 1$, de Adrián Ortiz, Eco milenario y Mitología, del maestro José Revelo Burbano, e, incluso, la obra aportada por el maestro Leonardo Coral, Ofrenda.
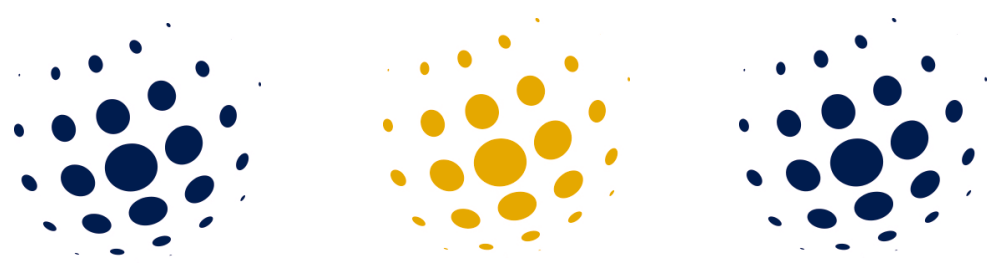


\section{Referencias}

Acuarimántima Trío (2017). Tríos americanos para clarinete, viola y piano. Ciudad de México. https://doi.org/10.18177/sym.2018.58.sr.11398

Alunno, M. (s.f.). Janus. Trío para clarinete, viola y piano. Medellín: el autor.

Arias, L. (1963). Elegía para clarinete, viola y piano. Buenos Aires: Editorial Argentina de Compositores.

A-Trio (2015a, 24 de febrero). ... YouTube. Recuperado el 10 de enero de 2018 de https://www.youtube.com/watch?v=6D4fqdscSuY

A-Trio (2015b, 24 de febrero). ... YouTube. Recuperado el 10 de enero de 2018, de https://www.youtube.com/watch?v=vMnA_rYOEtk

Cardona, A. (2001). La delgadina, variaciones para clarinete, viola y piano. San José de Costa Rica: Nuestra Cultura.

Castro Mora, C. J. (1989). El loco y la emperatriz en la recámara de los espejos. San José de Costa Rica: Urtext.

Centro de Investigación, Documentación e Información Musical Carlos Chávez, CEDINIM (2017). Centro de Investigación y Documentación Musical. Ciudad de México: Centro de Investigación, Documentación e Información Musical Carlos Chávez. Recuperado el 12 de septiembre de 2017 de www.cedinim.bellasartes.gob.mx

ClariPeru (2011). El clarinete en Latinoamérica. Biblioteca. Lima: ClariPeru. Recuperado el 10 de febrero de 2017 de http://blog.clariperu.org/p/biblioteca.html

Colegio de Compositores Latinoamericanos de Música de Arte (2012). Colegio de Compositores Latinoamericanos de Música de Arte. Ciudad de México: Colegio de Compositores Latinoamericanos de Música de Arte. Recuperado el 20 de enero de 2017 de http://www.colegiocompositores-la.org/

Coral, L. (2003). Ofrenda, para clarinete, viola y piano. Ciudad de México: el autor.

Correa Suárez, K. J. (2013). Viola latinoamericana. Medellín: Viola latinoamericana. Recuperado de violatinoamericana.blogspot.com.co

Delgado, S. (2016). Música concertante. San José de Costa Rica: el autor.

Escuela de Artes Musicales de Costa Rica (2016, 27 de septiembre). Música latinoamericana para clarinete, viola y piano. YouTube. Recuperado de https://www. youtube.com/watch?v=xA7rBMxNXco

Gandini, G. (1978-1980). Trioneiron, para clarinete, viola y piano. Buenos Aires: Editorial Argentina de Compositores. 
Gebr. Stark Musicverlag (2017). Gebr. Stark Musicverlag. Leipzig: Gebr. Stark Musicverlag. Recuperado el 7 de octubre de 2017 de www.stark-music.com

Miranda, R., y Tello, A. (2011). La música latinoamericana. La búsqueda perpetua: lo propio y lo universal de la cultura latinoamericana, vol. 4. Ciudad de México: Secretaría de Relaciones Exteriores, Dirección General del Acervo Histórico Diplomático.

Montano, M. A. (2016). Viola music from the Americas: music from Canada, United States, Cuba, Venezuela, Mexico y Brazil (disertación doctoral en Musical Arts, University of Maryland, College Park, MA). Recuperado de https://drum.lib.umd.edu/bitstream/ handle/1903/18322/Montano_umd_0117E_17072.pdf?sequence=1\&isAllowed=y

Mujeres en la Música (2017). Mujeres en la música. Madrid: Mujeres en la música. Recuperado el 18 de septiembre de 2017 de http://mujeresenlamusica.es

Nobre, M. (1978). La problemática de la música latinoamericana. Revista Musical Chilena, 32(142), 125-130. Recuperado de https://revistamusicalchilena.uchile.cl/ index.php/RMCH/article/view/611/515

Olivarec,J.I. (2000, septiembre-diciembre). La músicayla realidadlatinoamericana. Revista Cultura de Guatemala, III, 11-15.

Ortiz Rivera, A. (1994). Trío Nº1 para clarinete, viola y piano. San José de Costa Rica: el autor.

Pascual, J. (2008). Guía universal de la música clásica. Barcelona: Robinbook.

Pitombeira, L. (2010). Fantasía sobre "A muie rendêrá", Op 1f. Río de Janeiro: el autor.

Pitombeira, L. (2015a). Liduino Pitombeira. Recuperado el 3 de diciembre de 2017 de www.pitombeira.com

Pitombeira, L. (2015b). Praineiras Op. 200 para clarinete, viola y piano. Río de Janeiro: el autor.

Pitombeira, L. (2016). Brasiliana Op. 173c para clarinete, viola y piano. Río de Janeiro: el autor.

Pitombeira, L. (2017). Compositores de hoje: Trio Praineiras, intérprete, y S. R. Oliveira, dirección. Recuperado de https://open.spotify.com/user/kjohanaviola/ playlist/28VHlaDsJL3DWLiozxEDE8

Prieto Prieto, R. (2015). Referencias de libros para clarinete. Alcoy: Editorial Área de Innovación y Desarrollo. Recuperado de https://issuu.com/3ciencias/docs/ referencias_de_libros_para_clarinet

Quintana Moreno, H. J. (2005). Música europea y música latinoamericana del s XVIII. Revista de la Sociedad Venezolana de Musicología, ...(...), 133-166. Recuperado de http://www.academia.edu/11312891/M\%C3\%BAsica_europea_y_m\%C3\%BAsica_ latinoamericana_del_siglo_XVIII 
Revelo Burbano, J. (1998). Mitología. Adaptación para clarinete, viola y piano. San Juan de Pasto: el autor.

Revelo Burbano, J. (2002). Eco milenario, para clarinete, viola y piano. San Juan de Pasto: el autor.

Ripper, J. G. (2007). Trio para clarinete, viola y piano. Jeanné-Inc Publishing Co.

Ripper, J. G. (2017). João Guilherme Ripper. Recuperado el 3 de diciembre de 2017 de www.joaoripper.com.br

Román, D. (2016). LVTCN (Levitación) para clarinete (oboe), viola y Piano. San Juan, Puerto Rico: el autor.

Rubio, R. (2014). Música hondureña para el mundo. Recuperado el 7 de octubre de 2017 de http://rafrubio.blogspot.com.co/

Stephenson, C. D. (2011). Music for the viola from Latin America and Spain: canción y baile (disertación doctoral en Musical Arts, University of Maryland, College Park, MA). Recuperado de https://drum.lib.umd.edu/bitstream/handle/1903/12892/ DISSERTATION.pdf?sequence=1\&isAllowed=y

Tacuchian, R. (2017). A-Trio. Facebook. Recuperado el 25 de agosto de 2017 de https://www.facebook.com/A-Trio-1164521313659535/

Trío Contrastes (2016, 11 de septiembre). Brasiliana (L. Pitombeira). YouTube. Recuperado el 10 de enero de 2018 de https://www.youtube.com/watch?v=RZ01kUk3rtw

Vázquez, C. A. (2007). Cuatro sombras de una palma. San Juan, Puerto Rico: el autor.

Vázquez, C. A. (2017). Carlos Alberto Vázquez. tripod.com. Recuperado el 22 de julio de 2017 de carlosvazquez.tripod.com

Vdóvina Vdóvina, M. M. (2006). La viola en el conjunto instrumental y su desarrollo individualizado (disertación doctoral en Ciencias sobre Arte, Instituto Superior de Arte, Facultad de Música, La Habana). Recuperado de https://docplayer.es/7102137La-viola-en-el-conjunto-instrumental-y-su-desarrollo-individualizado.html

Víquez Córdoba, L. A. (2011). Buscando un por qué a los procesos de enseñanza y aprendizaje. Educación, 35(1), 1-20. Recuperado de http://www.redalyc.org/articulo. oa? id=44018789006 